\title{
PROLONGATIONS IN DIFFERENTIAL ALGEBRA
}

\author{
ERIC ROSEN
}

\begin{abstract}
We develop the theory of higher prolongations of algebraic varieties over fields in arbitrary characteristic with commuting Hasse-Schmidt derivations. Prolongations were introduced by Buium in the context of fields of characteristic 0 with a single derivation. Inspired by work of Vojta, we give a new construction of higher prolongations in a more general context. Generalizing a result of Buium in characteristic 0 , we prove that these prolongations are represented by a certain functor, which shows that they can be viewed as 'twisted jet spaces.' We give a new proof of a theorem of Moosa, Pillay, and Scanlon that the prolongation functor and jet space functor commute. We also prove that the $m^{t h}$-prolongation and $m^{t h}$-jet space of a variety are differentially isomorphic by showing that their infinite prolongations are isomorphic as schemes.
\end{abstract}

\section{INTRODUCTION}

Prolongations of algebraic varieties over a differential field of characteristic 0 were introduced by Buium [Bui92], and have also been considered in more general contexts [BV95, BV96, Sca97, MPS07]. The purpose of this paper is to develop the basic theory of prolongations of algebraic varieties over fields with finitely many commuting Hasse-Schmidt (or 'higher') derivations. Let us begin by describing the idea behind Buium's construction and the connection to jet spaces of varieties. We then describe the content of the paper in more detail.

Let $(K, \delta)$ be a differential field and let $R=K\left\{x_{1}, \ldots, x_{m}\right\}$ be the ring of differential polynomials, which is the polynomial ring in the infinitely many variables $\delta^{n} x_{i}, 0 \leq n$ and $i \leq m$. We say that $f \in R$ has order $\leq n$ if for every variable $\delta^{j} x_{i}$ that occurs in $f$, one has $j \leq n$. Observe that the set of elements of order $\leq n$ is a subring of $R$. Elements of $R$ can be viewed as functions on affine $m$-space $\mathbb{A}^{m}=K^{m}$ in a natural way.

More generally, let $X \subseteq \mathbb{A}^{m}$ be an affine variety over $(K, \delta)$ and let $A$ be the ring of regular functions on $X$. As above, there is a natural way to define the ring of differential polynomial functions of order $\leq n$ on $X$, called the $n^{t h}$-prolongation of $A$, which in this introduction we will denote $A^{(n)}$. Likewise, the $n^{t h}$-prolongation of $X$ is $P_{n}(X)=\operatorname{Spec} A^{(n)}$. Thus, there is a bijection between differential polynomial functions on $X$ of order $\leq n$ and regular functions on $P_{n}(X)$. For each $n$, there is a natural 'projection function' $\pi_{n}: P_{n}(X) \rightarrow X$, as well as a differential polynomial map $\nabla_{n}: X \rightarrow P_{n}(X)$, which is a section of $\pi_{n}$. In local coordinates, given $\bar{a} \in X$, $\nabla_{n}(\bar{a})=\left(\bar{a}, \delta(\bar{a}), \ldots, \delta^{n}(\bar{a})\right)$. Any differential polynomial function of order $\leq n$ on $X$ factors as the composition of $\nabla_{n}$ with a regular function on $P_{n}(X)$.

One can also define the infinite prolongation $A^{(\infty)}$ of $A$ as the ring of all differential polynomial functions on $X$, and the infinite prolongation $P_{\infty}(X)$ as Spec $A^{(\infty)}$. In this case, $A^{(\infty)}$ is naturally a differential ring, so that $X^{(\infty)}$ will be what Buium calls a $\mathcal{D}$-scheme, that is, a scheme over a differential field, equipped with a sheaf of differential rings. Note also that $P_{\infty}(X)$ is a pro-algebraic variety $P_{\infty}(X)=\lim _{\longleftarrow} P_{m}(X)$. As usual, everything above globalizes to arbitrary varieties.

Date: July 16, 2018.

This paper constitutes part of my dissertation, written at the University of Illinois at Chicago. I would like to thank my advisor, David Marker, for his guidance and support, and Lawrence Ein and Henri Gillet for helpful discussions on this material. I am also grateful to the referee for a careful reading of the paper and many detailed comments that have greatly improved the presentation. 
In an appendix to Bui93, Buium notes that prolongations are closely related to jet (or arc) spaces of varieties, which have been studied extensively in recent years (for example, [DL99, Cra04]). Recall that the $K$-valued points of the $n^{\text {th }}$-jet space of a variety are the $K[z] / z^{n+1}$-valued points of the variety itself. Alternatively, the $n^{\text {th }}$-jet space represents a certain functor, implicit in the above characterization. What Buium observed was that his prolongations represent a twisted version of this functor. Further, given a variety that descends to the field of constants, its $n^{\text {th }}$-prolongation is isomorphic to its $n^{\text {th }}$-jet space. The connection between jets and prolongations does not play a large role in Buium's theory, but it is central to the present work.

Below, we develop the theory of prolongations in a rather different way than Buium does, who built on earlier work of Johnson [Joh85]. Our approach was inspired by Votja [Voj06], who gives an elegant construction of jet spaces using higher derivations. The starting point for this paper was the observation that, over a differential field, one can modify Vojta's idea so as to define prolongations in a similar manner. This perhaps further clarifies the relation between jets and prolongations. It also leads to a rather direct proof that prolongations represent the twisted jet functor introduced by Buium. We should also mention Gillet's paper [Gil02], where he develops the theory of prolongations using adjoint functors, which allows him to give new proofs of earlier results of Buium and Kolchin.

Our work was also motivated by a recent paper of Moosa, Pillay, and Scanlon [MPS07] on the model theory of differentially closed fields in characteristic 0 with finitely many commuting derivations. In that paper, the prolongation of an algebraic variety is actually defined in terms of the twisted jet functor. The authors then go on to define more generally prolongations of differential algebraic varieties, which are not treated here. We hope that our paper could be read helpfully as a companion to theirs. Good references for the model theory of differential fields include Mar06, Pil02, [Sca02]. For applications to diophantine geometry, see, for example, [HP00, PZ03, Pil04].

Let us also say something about higher derivations. These are defined below, but the basic idea is that a ring can be equipped with a sequence of additive maps, $\left(D_{0}, D_{1}, \ldots, D_{m}, \ldots\right)$, with $D_{1}$ a derivation and each $D_{m}$ something like the $m^{\text {th }}$ derivative. In characteristic 0 , they are essentially equivalent to ordinary derivations, but in positive characteristic, higher derivations are more general and rather natural to use, for example, for developing differential Galois theory MvdP03. From a technical point of view, also, it was more straightforward to adapt Vojta's construction to fields equipped with higher derivations.

Summary of results. In Section 1, we begin by recalling the definition of a higher derivation (of order $m$ ) from an $R$-algebra $A$ to an $R$-algebra $B$. We then introduce the notion of a higher derivation over a differential ring $(R, \underline{D})$, and show that there is a universal object $\operatorname{HS}_{A /(R, \underline{D})}^{m}$, which is analogous to the module of Kähler differentials $\Omega_{A / R}$ in the usual case. This is the $m^{t h}$ prolongation of $A$, and we establish some basic properties of it. (In the first three sections, we restrict our attention to fields with a single higher derivation. In Section 4, we explain more briefly how to generalize our results to fields with commuting derivations.)

In Section 2, we define the $m^{t h}$-prolongation of a variety, which is by this point straightforward. We then prove a characterization of prolongations in terms of representable functors, a result due to Buium in characteristic 0. We also give a new proof of Moosa, Pillay, and Scanlon's theorem that the jet space functors and prolongation space functors commute.

In Section 3, we develop the foundations of Buium's theory of $\mathcal{D}$-schemes in arbitrary characteristic. Here we also prove the main result of this paper, that the $m^{\text {th }}$-prolongation of a variety is isomorphic to its $m^{\text {th }}$-jet space. Previously, this had only been known for $m=1$, where the $1^{\text {st }}$-jet space is just the tangent variety.

Finally, in the last section of the paper, we develop the theory of prolongations over fields with commuting derivations, very much following the treatment in the earlier sections of the paper. 
Conventions. Let $\mathbb{N}=\{0,1,2, \ldots\}$ denote the set of natural numbers, and $\mathbb{N}^{+}=\mathbb{N} \backslash\{0\}$. Throughout the paper, the variable $m$ will range over the ordered set $\mathbb{N} \cup\{\infty\}$, with $n<\infty$, for all $n \in \mathbb{N}$. Variables $i, j, k, l$ will range over $\mathbb{N}$. We frequently write, for example, $i \leq m$, as shorthand for $i \leq m$, if $m \in \mathbb{N}$, and $i<m$, if $m=\infty$. Likewise, $i=0, \ldots, m$ should be taken to mean exactly that, if $m \in \mathbb{N}$, and to mean $i=0,1, \ldots$, if $m=\infty$. We hope that such shorthand will not lead to any unclarity in the presentation.

All rings are commutative with unit.

\section{Higher DeRivations}

Definition 1.1. (See Mat89] or [Voj06.) Let $R$ be a ring, $f: R \rightarrow A$ and $R \rightarrow B$ be $R$ algebras, and $m \in \mathbb{N} \cup\{\infty\}$. A higher derivation of order $m$ from $A$ to $B$ over $R$ is a sequence $\underline{D}=\left(D_{0}, \ldots, D_{m}\right)$, or $\left(D_{0}, D_{1}, \ldots\right)$ if $m=\infty$, where $D_{0}: A \rightarrow B$ is an $R$-algebra homomorphism and $D_{1}, \ldots, D_{m}: A \rightarrow B$ are homomorphisms of (additive) abelian groups such that

(1) $D_{i}(f(r))=0$ for all $r \in R$ and $i \geq 1$;

(2) (Leibniz Rule) for all $a, b \in A$ and $k \leq m$,

$$
D_{k}(a b)=\sum_{i+j=k} D_{i}(a) D_{j}(b)
$$

Let $\operatorname{Der}_{R}^{m}(A, B)$ denote the set of such derivations.

Higher derivations are also called Hasse-Schmidt derivations.

Instead of condition (1), Matsumura requires the $D_{i}$ to be $R$-module homomorphisms, which is equivalent. Below, we will write $\left(D_{0}, \ldots, D_{m}\right)$, etc. even when $m=\infty$.

Example 1.2. With $R, A, B$, and $m$ as above, if $\mathbb{Q} \subseteq B \subseteq A$ and $D$ is a usual derivation from $A$ to $B$ over $R$, then $D_{i}=\frac{1}{i !} D^{i}, i \leq m$, is a higher derivation.

This is a relative notion of higher derivation. Viewing rings $A$ and $B$ as $\mathbb{Z}$-algebras, one also gets an absolute notion. Write $\operatorname{Der}^{m}(A, B)$ for $\operatorname{Der}_{\mathbb{Z}}^{m}(A, B)$.

Remark 1.3. Let $R, A, B$, and $m$ be as above, and $\underline{D}=\left(D_{0}, \ldots, D_{m}\right)$ be a sequence of maps from $A$ to $B$. There is an equivalent condition for $\underline{D}$ to be a higher derivation that will be useful below.

For $m<\infty$, let $B_{m}=B[t] /\left(t^{m+1}\right)$, the truncated polynomial ring, and for $m=\infty$, let $B_{m}=$ $B[[t]]$, the ring of power series. It is easy to check that $\underline{D}$ is a higher derivation if and only if the $\operatorname{map} g: A \rightarrow B_{m}$,

$$
a \mapsto D_{0}(a)+D_{1}(a) t+\ldots+D_{m}(a) t^{m}
$$

is a homomorphism of $R$-algebras.

In the special case $R=\mathbb{C}, A=\mathbb{C}(z), B=\mathbb{C}$, and $D_{i}=\frac{1}{i !} d^{i} / d z$, this says that the map taking a function $f(z) \in A$ to its (truncated) Taylor series expansion around 0 is a $\mathbb{C}$-algebra homomorphism.

Observe that $\operatorname{Der}_{R}^{m}(A,-)$ is a covariant functor from ( $R$-algebras) to (Sets), and is represented by a (graded) $R$-algebra that Vojta calls $\mathrm{HS}_{A / R}^{m}$, which is also an $A$-algebra. (See also Remark 1.10.(1) below.) For $m=1, \mathrm{HS}_{A / R}^{m}$ is just the symmetric algebra on $\Omega_{A / R}$.

Definition 1.4. Let $A$ be an $R$-algebra. A higher derivation on $A$ is a sequence of maps $\underline{D} \in$ $\operatorname{Der}_{R}^{\infty}(A, A)$ such that $D_{0}=\operatorname{Id}_{A}$. In this case, we call $(A, \underline{D})$ a $\mathcal{D}$-ring over $R$. A homomorphism $f:(A, \underline{D}) \rightarrow(B, \underline{D})$ between $\mathcal{D}$-rings over $R$ is an $R$-algebra homomorphism such that $f\left(D_{i}(a)\right)=$ $D_{i}(f(a))$, for all $a \in A$ and all $i$. We will often be interested in the case when $A$ is just a ring (that is, a $\mathbb{Z}$-algebra) and call $(A, \underline{D})$ simply a $\mathcal{D}$-ring. The set of constants of $(A, \underline{D})$ are those $a \in A$ such that $D_{i} a=0$, for all $i \geq 1$. Given $(A, \underline{D})$, say that $\underline{D}$ is iterative if for all $i, j, D_{i} \circ D_{j}=\left(\begin{array}{c}i+j \\ j\end{array}\right) D_{i+j}$. 
Below we will only consider iterative $\mathcal{D}$-rings. Note that it does not make sense to talk of a higher derivation from one ring to another being iterative.

Remark 1.5. As above, let $A_{m}=A[t] /\left(t^{m+1}\right)$, for $m<\infty$, and $A_{\infty}=A[[t]]$. Let $h: A_{m} \rightarrow A$ be the homomorphism sending $f(t) \in A_{m}$ to $f(0) \in A$. Then a sequence of maps $\underline{D}=\left(D_{0}, \ldots, D_{m}\right)$ from $A$ to $A$ is a higher derivation if and only if the map $d: A \rightarrow A_{m}$, with $d(a)=\sum_{i \leq m} D_{i}(a) t^{i}$, is a homomorphism and $h \circ d=\operatorname{Id}_{A}$.

Derivations on a ring extend uniquely to localizations (see [Mat89], Theorem 27.2, or [Oku87], Section 1.6, Theorem 1).

Lemma 1.6 (Quotient Rule). Let $(A, \underline{D})$ be a D-ring. For all invertible $b \in A$, and $n \in \mathbb{N}^{+}$,

$$
D_{n}\left(\frac{1}{b}\right)=\frac{-1}{b}\left(\sum_{i<n} D_{i}(b) \cdot D_{n-i}\left(\frac{1}{b}\right)\right) \text {. }
$$

To obtain this, observe $\left.0=D_{n}(1)=D_{n}\left(b \cdot \frac{1}{b}\right)=\sum_{i \leq n} D_{i}(b) \cdot D_{n-i}\left(\frac{1}{b}\right)\right)$, and solve for $D_{n}\left(\frac{1}{b}\right)$.

Lemma 1.7. Let $(R, \underline{D})$ be a $\mathcal{D}$-ring, and $S$ a multiplicative subset of $R$. Then there is a unique extension of $\underline{D}$ to $S^{-1} R$.

We now introduce higher derivations on $R$-algebras when $(R, \underline{D})$ is also a $\mathcal{D}$-ring. This is closely related to Buium's prolongations, where $(R, \delta)$ is a differential ring, $A$ is an $R$-algebra, and one considers derivations on $A$ that are 'compatible' with $\delta$.

Definition 1.8. Let $(R, \underline{D})$ be a $\mathcal{D}$-ring. An $R$-algebra $A$, given by $f: R \rightarrow A$, is an $(R, \underline{D})$-algebra if for all $r \in R$ and all $i, f(r)=0$ implies $f\left(D_{i}(r)\right)=0$; in other words, $\operatorname{Ker}(f)$ is a $\mathcal{D}$-ideal.

Let $f: R \rightarrow A$ and $B$ be $(R, \underline{D})$-algebras. A higher derivation from $A$ to $B$ of order $m$ over $(R, \underline{D})$ is a sequence $\underline{\delta}=\left(\delta_{0}, \ldots, \delta_{m}\right)$ such that $\delta_{0}: A \rightarrow B$ is an $R$-algebra homomorphism, $\delta_{i}: A \rightarrow B, 1 \leq i \leq m$, are (additive) abelian group homomorphisms, and

(1) $\delta_{i}(f(r))=\delta_{0}\left(f\left(D_{i}(r)\right)\right)$, for $r \in R$;

(2) (Leibniz Rule) $\delta_{k}(a b)=\sum_{i+j=k} \delta_{i}(a) \delta_{j}(b)$, for $a, b \in A$.

Let $\operatorname{Der}_{(R, \underline{D})}^{m}(A, B)$ denote the set of such derivations.

Note that if $(R, \underline{D})$ is trivial, that is, $D_{0}=\operatorname{Id}_{R}$ and $D_{i}=0, i \geq 1$, then this reduces to Definition 1.1,

As above, given an $(R, \underline{D})$-algebra $A, \operatorname{Der}_{(R, \underline{D})}^{m}(A,-)$ is a covariant functor from $((R, \underline{D})$-algebras $)$ to (Sets), which we will now observe to be representable.

Definition 1.9. Let $(R, \underline{D})$ be a $\mathcal{D}$-ring, $f: R \rightarrow A$ an $(R, \underline{D})$-algebra. For all $m$, define $\operatorname{HS}_{A /(R, \underline{D})}^{m}$ to be the $A$-algebra that is the quotient of the polynomial algebra $A\left[x^{(i)}\right]_{x \in A, 1 \leq i \leq m}$ by the ideal $I$ generated by:

(1) $(x+y)^{(i)}-x^{(i)}-y^{(i)}: x, y \in A, i=1, \ldots, m$;

(2) $(x y)^{(k)}-\sum_{i+j=k} x^{(i)} y^{(j)}: x, y \in A, k=1, \ldots, m$;

(3) $f(r)^{(i)}-f\left(D_{i}(r)\right): r \in R, i=1, \ldots, m$.

In $A\left[x^{(i)}\right]$, we identify $x \in A$ with $x^{(0)}$. There is a universal derivation $\underline{d}=\left(d_{0}, \ldots, d_{m}\right): A \rightarrow$ $\mathrm{HS}_{A /(R, \underline{D})}^{m}$ such that for $i \leq m$ and $x \in A, d_{i}(x)=x^{(i)}$.

Remarks 1.10. (1) With the above notation, if $(R, \underline{D})$ is a trivial $\mathcal{D}$-ring, then $\mathrm{HS}_{A /(R, \underline{D})}^{m}$ is the same as Vojta's $\mathrm{HS}_{A / R}^{m}$. In general, though, $\operatorname{HS}_{A /(R, \underline{D})}^{m}$ is not naturally graded, because of condition (3).

(2) For $m=1$, we get the first prolongation in the sense of Buium. 
(3) For $0 \leq m<n \leq \infty$, there are natural $A$-algebra homomorphisms $f_{m n}: \operatorname{HS}_{A /(R, \underline{D})}^{m} \rightarrow$ $\mathrm{HS}_{A /(R, \underline{D})}^{n}$. These form a directed system, and

$$
\mathrm{HS}_{A /(R, \underline{D})}^{\infty}=\lim _{i \in \mathbb{N}} \operatorname{HS}_{A /(R, \underline{D})}^{i}
$$

Definition 1.11. Let $(R, \underline{D})$ be a $\mathcal{D}$-ring. A $\mathcal{D}$ - $(R, \underline{D})$-algebra is a $\mathcal{D}$-ring $(A, \underline{D})$ that is also an $(R, \underline{D})$-algebra via some map $f: R \rightarrow A$, such that the derivation on $A$ is compatible with that on $R$. That is, for all $r \in R$ and all $i, D_{i}(f(r))=f\left(D_{i}(r)\right)$.

Lemma 1.12. Given an $(R, \underline{D})$-algebra $A$, there is a canonical way to make $\operatorname{HS}_{A /(R, \underline{D})}^{\infty}$ into a $\mathcal{D}-(R, \underline{D})$-algebra.

Proof. Extend the universal derivation $\underline{d}: A \rightarrow \operatorname{HS}_{A /(R, \underline{D})}^{\infty}$ to an (iterative) higher derivation on $\mathrm{HS}_{A /(R, \underline{D})}^{\infty}$ by setting

$$
d_{i}\left(x^{(j)}\right)=\left(\begin{array}{c}
i+j \\
i
\end{array}\right) x^{(i+j)}
$$

Definition 1.13. Let $(R, \underline{D})$ be a $\mathcal{D}$-ring. Let

$$
R_{m}= \begin{cases}R[t] /\left(t^{m+1}\right) & \text { for } m<\infty \\ R[[t]] & \text { for } m=\infty\end{cases}
$$

For each $m$, we define a 'twisted' homomorphism $e: R \rightarrow R_{m}$ by $e(r)=D_{0}(r)+D_{1}(r) t+\ldots+$ $D_{m}(r) t^{m}$. Let $\tilde{R}_{m}$ be the $R$-algebra isomorphic to $R_{m}$ as a ring, and made into an $R$-algebra via the map $e: R \rightarrow R_{m}$.

Let $f: R \rightarrow B$ be an $(R, \underline{D})$-algebra. Define $B_{m}=B[t] /\left(t^{m+1}\right)$, for $m<\infty$, and $B_{\infty}=B[[t]]$. Let $\tilde{B}_{m}$ be the ring $B_{m}$ made into an $R$-algebra via the map $\tilde{f}: R \rightarrow \tilde{B}_{m}$ that sends

$$
r \mapsto f\left(D_{0}(r)\right)+f\left(D_{1}(r)\right) t+\ldots+f\left(D_{m}(r)\right) t^{m} .
$$

Proposition 1.14. Let $(R, \underline{D})$ be a $\mathcal{D}$-ring. For all $m, R_{m}$ and $\tilde{R}_{m}$ are isomorphic as $R$-algebras.

Proof. Suppose first that $m<\infty$. We claim that the map $\psi: R_{m} \rightarrow \tilde{R}_{m}$ with $\psi(r)=e(r)=$ $D_{0}(r)+D_{1}(r) t+\ldots+D_{m}(r) t^{m}$, for $r \in R$, and $\psi(t)=t$ is an isomorphism of $R$-algebras. Clearly, $\psi$ is a homomorphism, so it suffices to check that it is injective and surjective.

Let $a=a_{0}+a_{1} t+\ldots+a_{m} t^{m}$, so $\psi(a)=e\left(a_{0}\right)+e\left(a_{1}\right) t+\ldots+e\left(a_{m}\right) t^{m}$. Rearranging terms, one gets

$$
\psi(a)=a_{0}+\left(D_{1}\left(a_{0}\right)+a_{1}\right) t+\left(D_{2}\left(a_{0}\right)+D_{1}\left(a_{1}\right)+a_{2}\right) t^{2}+\cdots+\left(D_{m}\left(a_{0}\right)+\ldots+a_{m}\right) t^{m} .
$$

Suppose that $\psi(a)=0$, so in particular each coefficient of $\psi(a)$ as a polynomial in $t$ is 0 . Thus, $a_{0}=0$. Looking at the next term, $0=D_{1}\left(a_{0}\right)+a_{1}=a_{1}$. Continuing this way, one sees that all of the $a_{i}$ 's are 0 , so $a$ itself is 0 and $\psi$ is injective.

To show that $\psi$ is surjective, it suffices to show that for each $r \in R, r=r+0 t+\ldots+0 t^{m} \in \tilde{R}_{m}$ is in $\operatorname{Im}(\psi)$. (Of course, $r \neq \psi(r)$.) For fixed $r$, we iteratively define a sequence, $c_{0}, c_{1}, \ldots, c_{m}$, of elements of $R_{m}$ with the following properties. One, for all $i \leq m$, the constant term of $\psi\left(c_{i}\right)$, as a polynomial in $t$, is $r$. Two, for $i \geq 1$, and $1 \leq j \leq i$, the coefficient of $t^{j}$ in $\psi\left(c_{i}\right)$ is 0 . Then $\psi\left(c_{m}\right)=r$, as desired. Set $c_{0}=r$. For the iterative step, suppose that $c_{0}, \ldots, c_{i}$ have been defined, and that $\psi\left(c_{i}\right)=r+a_{i+1} t^{i+1}+\ldots+a_{m} t^{m}$. Let $c_{i+1}=c_{i}-a_{i+1} t^{i+1}$. Clearly, this procedure yields such a sequence.

For $m=\infty$, given the isomorphisms $\psi_{i}: R_{i} \rightarrow \tilde{R}_{i}, i<\infty$, it suffices to note that $R_{\infty}$ and $\tilde{R}_{\infty}$ are the inverse limits of $\left\{R_{i}\right\}_{i<\infty}$ and $\left\{\tilde{R}_{i}\right\}_{i<\infty}$, respectively. The required isomorphism $\psi_{\infty}: R_{\infty} \rightarrow \tilde{R}_{\infty}$ is again given by sending $r \in R$ to $e(r)$, and sending $t$ to $t$. 
More generally, we have the following.

Proposition 1.15. Let $(R, \underline{D})$ be a $\mathcal{D}$-ring, and $B$ an $(R, \underline{D})$-algebra such that $\underline{D}$ extends to a derivation on $B$. Then $\tilde{B}_{m} \cong B_{m}$, as $R$-algebras.

Proof. Choose a derivation $\underline{D}$ on $B$ extending $(R, \underline{D})$, and then argue as above.

For fields of characteristic 0 , any (higher) derivation on a field $K$ can be extended to a derivation on any extension field $L \supseteq K$, so one has the following corollary.

Corollary 1.16. Let $(K, \underline{D})$ be a $\mathcal{D}$-field of characteristic 0 and $L \supseteq K$ an extension field. Then $\tilde{L}_{m} \cong L_{m}$, as $K$-algebras.

On the other hand, $L_{m}$ and $\tilde{L}_{m}$ are not always isomorphic.

Proposition 1.17. Let $(K, \underline{D})$ be a $\mathcal{D}$-field of characteristic $p>0$, and let $L$ be a purely inseparable algebraic extension of $K$, such that there is an $a \in L, b \in K$, with $a^{p}=b$ and $D_{1}(b) \neq 0$. Then for all $m \geq 1, L_{m}$ and $\tilde{L}_{m}$ are not isomorphic as $K$-algebras.

Proof. We show that there is no $K$-algebra homomorphism from $L$ to $\tilde{L}_{m}$, which immediately implies the proposition. In particular, we argue that any such homomorphism would give an extension of $D_{1}$ to a derivation on $L$, which is impossible (as $0 \neq D_{1}(b)=D_{1}\left(a^{p}\right)=p a^{p-1} D_{1}(a)=0$, contradiction).

Suppose that $\phi: L \rightarrow \tilde{L}_{m}$ is a $K$-algebra homomorphism. For all $c \in K, \phi(c)=D_{0}(c)+D_{1}(c) t+$ $\ldots+D_{m}(c) t^{m}$. For $x \in L$, write $\phi(x)=\phi_{0}(x)+\phi_{1}(x) t+\ldots+\phi_{m}(x) t^{m}$, with $\phi_{i}: L \rightarrow L$, for $i=0, \ldots, m$. We claim that for all $x \in L, \phi_{0}(x)=x$. Indeed, this is clear for $x \in K$, as $\phi_{0}(x)=D_{0}(x)=x$. Otherwise, $x^{p^{n}}=y$, for some $n$ and some $y \in K$. Then

$$
\phi(x)^{p^{n}}=\left(\phi_{0}(x)+\phi_{1}(x) t+\ldots+\phi_{m}(x) t^{m}\right)^{p^{n}}=\phi_{0}(x)^{p^{n}}+t \cdot g(t)
$$

and also

$$
\phi(x)^{p^{n}}=\phi\left(x^{p^{n}}\right)=\phi(y)=D_{0}(y)+t \cdot h(t)
$$

with $g(t), h(t)$ polynomials in $L[t]$. Thus $\phi_{0}(x)^{p^{n}}=D_{0}(y)=y$, so $\phi_{0}(x)=x$, as desired.

By the claim, $(L, \phi)$ is a higher derivation of order $m$ that extends $(K, \underline{D})$. In particular, $\phi_{1}$ is an extension of $D_{1}$ to $L$, which is impossible.

Proposition 1.18. Let $(R, \underline{D})$ be a D-ring, $R \rightarrow A$ and $R \rightarrow B$ be $(R, \underline{D})$-algebras. Given a higher derivation $\underline{\delta}=\left(\delta_{0}, \ldots, \delta_{m}\right): A \rightarrow B$ there exists a unique $(R, \underline{D})$-algebra homomorphism, $\phi: \mathrm{HS}_{A /(R, \underline{D})}^{m} \rightarrow B$ such that $\left(\delta_{0}, \ldots, \delta_{m}\right)=\left(\phi \circ d_{0}, \ldots, \phi \circ d_{m}\right)$. Thus $\mathrm{HS}_{A /(R, \underline{D})}^{m}$ (together with the universal derivation $\left.\underline{d}: A \rightarrow \operatorname{HS}_{A /(R, \underline{D})}^{m}\right)$ represents the functor $\operatorname{Der}_{(R, \underline{D})}^{m}(A,-)$.

Proof. Define $\phi_{0}: A\left[x^{(i)}\right]_{x \in A, i=1, \ldots, m} \rightarrow B$ by $x^{(i)} \mapsto \delta_{i}(x)$. By the construction of the ideal $I \subseteq A\left[x^{(i)}\right]$ and properties of derivation, we get that $\operatorname{Ker}\left(\phi_{0}\right) \supseteq I$, so there is an induced map $\phi: \mathrm{HS}_{A /(R, \underline{D})}^{m} \rightarrow R$. As $\underline{\delta}=\phi \circ \underline{d}, \phi$ is unique. Thus the map

is bijective.

$$
\operatorname{Hom}_{R}\left(\operatorname{HS}_{A /(R, \underline{D})}^{m}, B\right) \longrightarrow \operatorname{Der}_{(R, \underline{D})}^{m}(A, B)
$$

Compare the following proposition to Remark 1.3 ,

Proposition 1.19. Let $(R, \underline{D})$ be a D-ring, $f: R \rightarrow A, g: R \rightarrow B$ be $(R, \underline{D})$-algebras. Given a derivation $\underline{\delta}=\left(\delta_{0}, \ldots, \delta_{m}\right) \in \operatorname{Der}_{(R, \underline{D})}^{m}(A, B)$, define a map $\phi=\phi_{\underline{\delta}}: A \rightarrow \tilde{B}_{m}$ by $\phi(a)=$ $\delta_{0}(a)+\delta_{1}(a) t+\ldots+\delta_{m}(x) t^{m}$. Then $\phi_{\underline{\delta}} \in \operatorname{Hom}_{R}\left(A, \tilde{B}_{m}\right)$ and the map

$$
\underline{\delta} \mapsto \phi_{\underline{\delta}}: \operatorname{Der}_{(R, \underline{D})}^{m}(A, B) \rightarrow \operatorname{Hom}_{R}\left(A, \tilde{B}_{m}\right)
$$

is a bijection. 
Proof. The $\delta_{i}$ are homomorphism of the additive groups, so $\phi$ is also. The Leibniz Rule implies that $\phi$ is multiplicative, so it only remains to show that $\phi \circ f=\tilde{g}$, where $\tilde{g}: R \rightarrow \tilde{B}_{m}$ is the homomorphism that makes $\tilde{B}_{m}$ into an $R$-algebra. Check,

$$
\begin{aligned}
\phi \circ f(x) & =\delta_{0}(f(x))+\delta_{1}(f(x)) t+\ldots+\delta_{m}(f(x)) t^{m} \\
& =\delta_{0}(f(x))+\delta_{0}\left(f\left(D_{1}(x)\right)\right) t+\ldots+\delta_{0}\left(f\left(D_{m}(x)\right)\right) t^{m} \\
& =g(x)+g\left(D_{1}(x)\right) t+\ldots+g\left(D_{m}(x)\right) t^{m} \\
& =\tilde{g}(x)
\end{aligned}
$$

This establishes injectivity. To show surjectivity, we just reverse the direction of the argument. Suppose that $h: A \rightarrow \tilde{B}_{m}$ is an $R$-algebra homomorphism, which we can write as $h(a)=h_{0}(a)+$ $h_{1}(a) t+\ldots+h_{m}(a) t^{m}$, each $h_{i}$ a map from $A$ to $B$. We claim that $\left\{h_{i}: i \leq m\right\}$ is a higher derivation from $A$ to $B$. Clearly the $h_{i}$ are additive and satisfy the Leibniz Rule. So it suffices to show that for $r \in R$ and $i \leq m, h_{i}(f(r))=h_{0}\left(f\left(D_{i}(r)\right)\right)$. Since $h$ is an $R$-algebra homomorphism, one has $h_{i}(f(r))=h_{0}\left(f\left(D_{i}(r)\right)=g\left(D_{i}(r)\right)\right.$.

The next corollary follows immediately from Proposition 1.18 and Proposition 1.19, It is the main point in the characterization of prolongations in terms of representable functors.

Corollary 1.20 (Buium). There is a natural bijection

$$
\operatorname{Hom}_{R}\left(\mathrm{HS}_{A /(R, \underline{D})}^{m}, B\right) \longrightarrow \operatorname{Hom}_{R}\left(A, \tilde{B}_{m}\right)
$$

The next result is due to Buium Bui93 and Gillet Gil02 in a slightly different context. In fact, Gillet defines the prolongation functor to be the left adjoint of the forgetful functor from differential algebras to algebras.

Proposition 1.21. Let $(R, \underline{D})$ be a $\mathcal{D}$-ring, $\operatorname{Alg}_{R}$ be the category of $(R, \underline{D})$-algebras, and $\mathcal{D}-\mathrm{Alg}_{R}$ be the category of $\mathcal{D}-(R, \underline{D})$-algebras. Let $U$ be the forgetful functor $\mathcal{D}-\operatorname{Alg}_{R} \rightarrow \operatorname{Alg}_{R}$. Then the functor $F: \mathrm{Alg}_{R} \rightarrow \mathcal{D}-\mathrm{Alg}_{R}$, sending $A$ to $\mathrm{HS}_{A /(R, \underline{D})}^{\infty}$, is the left adjoint of $U$.

Proof. Essentially immediate from the explicit construction given of $\mathrm{HS}_{A /(R, \underline{D})}^{\infty}$. That is, given an $R$-algebra map $f: A \rightarrow\left(B, \underline{D}^{B}\right)$, there is an obvious, unique way to lift $f$ to a $\mathcal{D}$ - $(R, \underline{D})$-algebra map $f^{\infty}: \operatorname{HS}_{A /(R, \underline{D})}^{\infty} \rightarrow\left(B, \underline{D}^{B}\right)$. For example, for $x^{(i)} \in \mathrm{HS}_{A /(R, \underline{D})}^{\infty}, x \in A$, then $f^{\infty}\left(x^{(i)}\right)=$ $D_{i}^{B}(f(x))$.

The next result is what Vojta calls the second fundamental exact sequence, adapted to our context. For completeness, we include his proof, which carries over directly.

Proposition 1.22 (Second fundamental exact sequence). Let $(R, \underline{D})$ be a $\mathcal{D}$-ring and $R \rightarrow A \rightarrow B$ a sequence of ring homomorphisms. Assume that $A \rightarrow B$ is surjective, and let $I$ be its kernel. Let $J$ be the ideal in $\mathrm{HS}_{A /(R, \underline{D})}^{m}$ generated by $\left\{d_{i} x: i \leq m, x \in I\right\}$. Then the following sequence is exact.

$$
0 \longrightarrow J \longrightarrow \mathrm{HS}_{A /(R, \underline{D})}^{m} \longrightarrow \mathrm{HS}_{B /(R, \underline{D})}^{m} \longrightarrow 0
$$

In the definition of $J$, it suffices to let $x$ vary over a set of generators of $I$.

Proof. Exactness on the left is immediate. The natural map $h: \mathrm{HS}_{A /(R, \underline{D})}^{m} \longrightarrow \mathrm{HS}_{B /(R, \underline{D})}^{m}$ is surjective and its kernel contains $J$, so it remains to show that $\operatorname{Ker}(h)=J$. 
From the definition of $\mathrm{HS}^{m}$, we have the following commutative diagram.

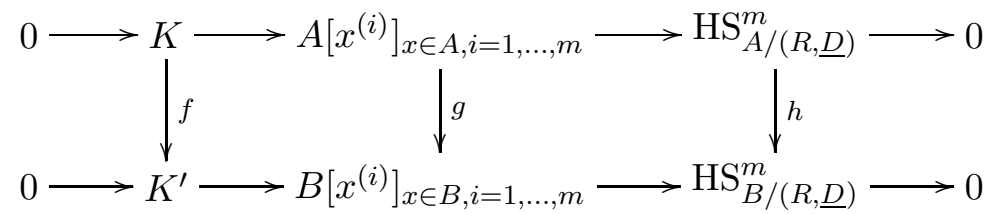

By Definition 1.9, the map $f$ is surjective, so by the Snake Lemma, $\operatorname{Ker}(g)$ maps onto $\operatorname{Ker}(h)$. But $\operatorname{Ker}(g)$ is generated by

$$
I \cup\left\{d_{i} x-d_{i} y: i=1, \ldots, m ; x, y \in A ; x-y \in I\right\} .
$$

This implies that the kernel of $h$ is generated by the set $\left\{d_{i} x: i=0, \ldots, m, x \in I\right\}$, as desired.

The next two results also occur in Vojta Voj06.

Proposition 1.23. Let $(R, \underline{D})$ be a $\mathcal{D}$-ring, and $A=R\left[x_{i}\right]_{i \in I}$. Then $\mathrm{HS}_{A /(R, \underline{D})}^{m}$ is the polynomial algebra $A\left[d_{j} x_{i}\right]_{i \in I, j=1, \ldots, m}$.

Proof. Essentially obvious, but also proved in Voj06.

Corollary 1.24. Let $A$ be an $(R, \underline{D})$-algebra, $A \cong R\left[x_{i}\right]_{i \in I} /\left(f_{j}\right)_{j \in J}$. Then

$$
\mathrm{HS}_{A /(R, \underline{D})}^{m} \cong A\left[d_{k} x_{i}\right]_{i \in I, k=1, \ldots, m} /\left(d_{k} f_{j}\right)_{j \in J, k=1, \ldots, m} .
$$

Suppose further that all of the coefficients of the polynomials $f_{j}, j \in J$, are constants in the ring $R$. Then $\mathrm{HS}_{A /(R, \underline{D})}^{m}$ is the same as $\mathrm{HS}_{A / R}^{m}$, as defined by Vojta.

Proof. The first statement follows from Propositions 1.22 and 1.23. The second follows from the first, and the analogous statement from Voj06.

\section{Prolongations}

In this section, we assume throughout that $(K, \underline{D})$ is a $\mathcal{D}$-field. Probably everything also works over $\mathcal{D}$-rings. We define prolongations of schemes/varieties over $(K, \underline{D})$. In characteristic 0 , we essentially get Buium's prolongations, though there is a slight difference since we are using higher derivations. The construction of the prolongations is direct, but the results of the previous section provide the connection with representable functors. In characteristic 0, this agrees with Buium, but in characteristic $p>0$, it avoids problems that would arise if one tries to adapt Buium directly to characteristic $p$, involving 'dividing by $p$.'

Lemma 2.1. Let $A$ be $a(K, \underline{D})$-algebra and and $S$ a multiplicative subset of $A$. Then there is an isomorphism

$$
\mathrm{HS}_{A /(K, \underline{D})}^{m} \otimes_{A} S^{-1} A \longrightarrow \mathrm{HS}_{S^{-1} A /(K, \underline{D})^{\prime}}^{m}
$$

Proof. For all $a \in A$, let $\bar{a}$ denote the image of $a$ in $S^{-1} A$ under the canonical map. We show that the natural map $\phi$ that sends $d_{i} a \otimes s^{-1} b$ to $s^{-1} b \cdot d_{i} \bar{a}$, for all $a, b \in A, s \in S$, and $i \leq m$, is an isomorphism. Clearly, $\phi$ is a homomorphism. By the quotient rule, for $i \leq m$ and $s^{-1} \bar{b} \in S^{-1} A, d_{i}\left(s^{-1} \bar{b}\right)$ can be written as $s^{-n} c$, for some $c \in \mathrm{HS}_{A /(K, \underline{D})}^{m}$, so $\phi$ is surjective. To show that $\phi$ is injective, it suffices to define its inverse. Let $s^{-1} \bar{b} \in S^{-1} A$. We want to define $\phi^{-1}\left(d_{i}\left(s^{-1} \bar{b}\right)\right)$ as $c \otimes s^{-n}$, but for this we need to check that if $s^{-1} \bar{b}=t^{-1} \bar{c}$ in $S^{-1} A$, then $\phi^{-1}\left(d_{i}\left(s^{-1} \bar{b}\right)\right)=\phi^{-1}\left(d_{i}\left(t^{-1} \bar{c}\right)\right)$, for all $i \leq m$. To simplify the presentation, let us assume that $s=t=1$. As $\bar{b}=\bar{c}$ in $S^{-1} A$ is equivalent to there being an $s \in S$ such that $s(c-b)=0$ in $A$, it suffices for us to show that for all $a \in A$, if $\bar{a}=0$ in $S^{-1} A$, that is, there is $s \in S$ such that $s a=0$ in $A$, then $d_{i} a \otimes 1=0$ in $\operatorname{HS}_{A /(K, \underline{D})}^{m} \otimes_{A} S^{-1} A$, for $i \leq m$. 
We argue by induction on $i$. The case $i=0$ is obvious, so assume that we have proved that $d_{j} a \otimes 1=0$ in $\operatorname{HS}_{A /(K, \underline{D})}^{m} \otimes_{A} S^{-1} A$, for all $j<i$. Then

$$
0=s a \otimes 1=d_{i}(s a) \otimes 1=\sum_{j+k=i}\left(d_{j} s d_{k} a \otimes 1\right)=s d_{i} a \otimes 1=\left(d_{i} a \otimes 1\right)(1 \otimes s),
$$

so $\left(d_{i} a \otimes 1\right)=0$ in $\operatorname{HS}_{A /(K, \underline{D})}^{m} \otimes_{A} S^{-1} A$, as desired.

The next theorem is the differential version of Theorem 4.3 of [Voj06], and is an easy consequence of Lemma 2.1, exactly as in Vojta.

Theorem 2.2. Let $X$ be a $K$-scheme. For all $m$, there exists a sheaf of $\mathcal{O}_{X}$-algebras $\operatorname{HS}_{X /(K, \underline{D})}^{m}$ such that (i) for each open affine $\operatorname{Spec} A \subseteq X$, there is an isomorphism

$$
\phi_{A}: \Gamma\left(\operatorname{Spec} A, \operatorname{HS}_{X /(K, \underline{D})}^{m}\right) \longrightarrow \operatorname{HS}_{A /(K, \underline{D})}^{m}
$$

of $(K, \underline{D})$-algebras, and (ii) the various $\phi_{A}$ are compatible with the localization isomorphism of Lemma 2.1. Moreover, the collection $\left(\left(\mathrm{HS}_{X /(K, \underline{D})}^{m}\right),\left(\phi_{A}\right)_{A}\right)$ is unique.

Definition 2.3. Let $X$ be a $K$-scheme. For all $m$, the $m^{\text {th }}$-prolongation of $X$ is the scheme

$$
P_{m}(X /(K, \underline{D})):=\operatorname{Spec} \operatorname{HS}_{X /(K, \underline{D})}^{m} .
$$

Suppose that $A$ is a $(K, \underline{D})$-algebra. We write $P_{m}(A /(K, \underline{D}))=P_{m}(\operatorname{Spec} A /(K, \underline{D}))$, which equals Spec $\operatorname{HS}_{A /(K, \underline{D})}^{m}$.

We will also write $X^{(m)}$ or $P_{m}(X)$ for $P_{m}(X /(K, \underline{D}))$.

(For the definition of Spec, see, for example, [Har77] Ch. II, Ex. 5.17.)

Recall that $K_{m}=K[t] /\left(t^{m+1}\right)$, for $m<\infty, K_{\infty}=K[[t]]$, and that $e: K \rightarrow K_{m}$ denotes the twisted homomorphism. We also let $e: \operatorname{Spec} K_{m} \rightarrow \operatorname{Spec} K$ denote the corresponding twisted morphism of schemes. Given a $K$-scheme $Y$, let $\left(Y \times{ }_{K} \operatorname{Spec} K_{m}\right)$ denote the scheme $\left(Y \times_{K} \operatorname{Spec} K_{m}\right)$ made into a $K$-scheme via the map $e \circ p:\left(Y \times_{K} \operatorname{Spec} K_{m}\right) \rightarrow \operatorname{Spec} K$, where $p:\left(Y \times_{K} \operatorname{Spec} K_{m}\right) \rightarrow$ Spec $K_{m}$ is the canonical projection.

Theorem 2.4 (Buium). Let $X$ be a $K$-scheme. For all $m$, the scheme $P_{m}(X)$ represents the functor from $K$-schemes to sets given by

$$
Y \mapsto \operatorname{Hom}_{K}\left(\left(Y \times_{K} \operatorname{Spec} K_{m}\right), X\right) .
$$

Proof. For $X$ and $Y$ affine, this follows immediately from Corollary 1.20, The general case follows by gluing affines.

Recall that, given a $K$-scheme $X$, the $m^{\text {th }}$-jet space of $X$, which we denote $J_{m}(X)$, is the scheme that represents the following functor from $K$-schemes to sets.

$$
Y \mapsto \operatorname{Hom}_{K}\left(Y \times_{K} \operatorname{Spec} K_{m}, X\right)
$$

Buium's theorem clarifies the relationship between prolongations and jets. One also has the following fact, due again to Buium.

Proposition 2.5. Let $X$ be a $(K, \underline{D})$-scheme such that $X=X^{\prime} \times_{C} \operatorname{Spec} K$, where $C$ is the field of constants of $K$, and $X^{\prime}$ is some $C$-scheme. (That is, $X$ descends to, or is defined over, $C$.) Then for all $m, P_{m}(X) \cong J_{m}(X)$.

Proof. This follows from Corollaries 1.24 and 2.10, below, and the description of jets in Voj06 (see, for example, Theorem 4.3 and Definition 4.4). 
The next result, due to Moosa, Pillay, and Scanlon, generalizes the well-known fact that for all $m, n \leq \infty, J_{m}\left(J_{n}(X)\right)=J_{n}\left(J_{m}(X)\right)$, which can be seen by observing that they represent the same functor. In the original version of [MPS07] it was stated without proof. A revised version contains a proof using the Weil restriction.

Theorem 2.6 (Moosa, Pillay, and Scanlon). Let $X$ be a $K$-scheme. For all $m, n \leq \infty$,

$$
J_{m}\left(P_{n}(X)\right) \cong P_{n}\left(J_{m}(X)\right) .
$$

Proof. We include two proofs. The first is direct and uses the construction of jets and prolongations from [Voj06] and this paper. The second, closer in spirit to [MPS07], shows that $J_{m}\left(P_{n}(X)\right)$ and $P_{n}\left(J_{m}(X)\right)$ represent the same functor.

It suffices to prove this for affine schemes, so assume that $X=\operatorname{Spec} A$. Even though $K$ is a differential field, we will use $\operatorname{HS}_{A / K}^{m}$ to denote the $A$-algebra defined by Vojta, which is defined exactly like $\operatorname{HS}_{A /(K, \underline{D})}^{m}$ in Definition [1.9, except that one replaces condition (3) with

$$
f(r)^{(i)}: r \in K, i=1, \ldots, m .
$$

The point from our perspective is that $\operatorname{Spec~HS}_{A / K}^{m}$ is the $m^{t h}$-jet space of $X$, while $\operatorname{Spec~HS}_{A /(K, \underline{D})}^{m}$ is the $m^{t h}$-prolongation of $X$. Thus

and

$$
J_{m}\left(P_{n}(X)\right)=\operatorname{Spec} \operatorname{HS}_{\mathrm{HS}_{A /(K, \underline{D})}^{n}}^{m} / K
$$

$$
P_{n}\left(J_{m}(X)\right)=\operatorname{Spec} \mathrm{HS}_{\mathrm{HS}_{A / K}^{m}}^{n} /(K, \underline{D})^{\prime}
$$

We want to show that the two $K$-algebras above are isomorphic. Let us use $\underline{d}$ for the universal derivation on $\mathrm{HS}^{m}$, corresponding to jets, and $\underline{\delta}$ for the universal derivation on $\bar{H}^{n}$, corresponding to prolongations. An arbitrary element of $\mathrm{HS}_{\mathrm{HS}_{A /(K, \underline{D})}^{m} / K}^{-}$can be written as a sum of terms

$$
d_{i} \delta_{j} a: \quad i \leq m, j \leq n, a \in A,
$$

and an arbitrary element of $\mathrm{HS}_{\mathrm{HS}_{A / K}^{m}}^{n} /(K, \underline{D})$ as a sum of terms

$$
\delta_{j} d_{i} a: \quad i \leq m, j \leq n, a \in A .
$$

We claim that the $K$-algebra morphism $\theta: \mathrm{HS}_{\mathrm{HS}_{A /(K, \underline{D})}^{n} / K}^{m} \rightarrow \mathrm{HS}_{\mathrm{HS}_{A / K}^{m} /(K, \underline{D})}^{n}$ with $\theta\left(d_{i} \delta_{j} a\right)=\delta_{j} d_{i} a$ is an isomorphism.

First we check that $\theta$ is well-defined. For example, $d_{i}\left(\delta_{j}(a+b)-\delta_{j}(a)-\delta_{j}(b)\right)=0$, so we check

$$
\theta\left(d_{i}\left(\delta_{j}(a+b)-\delta_{j}(a)-\delta_{j}(b)\right)\right)=\delta_{j}\left(d_{i}(a+b)-d_{i}(a)-d_{i}(b)\right)=0 .
$$

Likewise,

And we check

$$
\begin{aligned}
0 & =d_{i}\left(\delta_{j}(a b)-\sum_{k+l=j} \delta_{k}(a) \delta_{l}(b)\right. \\
& =d_{i} \delta_{j}(a b)-\sum_{k+l=j} d_{i}\left(\delta_{k}(a) \delta_{l}(b)\right) \\
& =d_{i} \delta_{j}(a b)-\sum_{k+l=j} \sum_{m+n=i} d_{m} \delta_{k}(a) d_{n} \delta_{l}(b) .
\end{aligned}
$$

$$
\begin{aligned}
& \theta\left(d_{i} \delta_{j}(a b)-\sum_{k+l=j} \sum_{m+n=i} d_{m} \delta_{k}(a) d_{n} \delta_{l}(b)\right) \\
&= \delta_{j} d_{i}(a b)-\sum_{m+n=i} \sum_{k+l=j} \delta_{k} d_{m}(a) \delta_{l} d_{n}(b) \\
&= \delta_{j} d_{i}(a b)-\sum_{m+n=i} \delta_{j}\left(d_{m}(a) d_{n}(b)\right) \\
&= \delta_{j}\left(d_{i}(a b)-\sum_{m+n=i} d_{m}(a) d_{n}(b)\right)=0 . \\
& 10
\end{aligned}
$$


Finally, for $c \in K$, we have $0=d_{i}\left(\delta_{j}(c)-\delta_{0} D_{j}(c)\right)$. For $i \geq 1$,

$$
\theta\left(d_{i}\left(\delta_{j}(c)-\delta_{0} D_{j}(c)\right)\right)=\delta_{j} d_{i}(c)-\delta_{0} d_{i}\left(D_{j}(c)\right)=0
$$

and, for $i=0$,

$$
\theta\left(d_{0}\left(\delta_{j}(c)-\delta_{0} D_{j}(c)\right)\right)=\delta_{j} d_{0}(c)-\delta_{0} d_{0}\left(D_{j}(c)\right)=\delta_{j}(c)-\delta_{0}\left(D_{j}(c)\right)=0 .
$$

Now that we know that $\theta$ is well-defined, it is clear from the definition that it respects sums and products. Finally, it is clearly a bijection, since there is an obvious inverse.

We now give a second proof, along the lines of [MPS07]. Let $Y$ be a $K$-scheme. There are natural bijections

and also natural bijections

$$
\begin{aligned}
& \operatorname{Hom}_{K}\left(Y, J_{m}\left(P_{n}(X)\right)\right) \\
\simeq & \operatorname{Hom}_{K}\left(Y \times_{K} \operatorname{Spec} K_{m}, P_{n}(X)\right) \\
\simeq & \operatorname{Hom}_{K}\left(\left(\left(Y \times_{K} \operatorname{Spec} K_{m}\right) \times_{K} \operatorname{Spec} K_{n}\right)^{\sim}, X\right)
\end{aligned}
$$

$$
\begin{aligned}
& \operatorname{Hom}_{K}\left(Y, P_{n}\left(J_{m}(X)\right)\right) \\
\simeq & \operatorname{Hom}_{K}\left(\left(Y \times_{K} \operatorname{Spec} K_{n}\right)^{\sim}, J_{m}(X)\right) \\
\simeq & \operatorname{Hom}_{K}\left(\left(\left(Y \times_{K} \operatorname{Spec} K_{n}\right)^{\sim} \times_{K} \operatorname{Spec} K_{m}\right), X\right)
\end{aligned}
$$

Thus, it suffices to show that for all $Y$,

$$
\left(\left(Y \times_{K} \operatorname{Spec} K_{m}\right) \times_{K} \operatorname{Spec} K_{n}\right)^{\sim} \cong\left(\left(Y \times_{K} \operatorname{Spec} K_{n}\right)^{\sim} \times_{K} \operatorname{Spec} K_{m}\right) .
$$

In fact, it suffices to prove this for $Y$ affine. We rephrase this as a question about isomorphisms of $K$-algebras. Given a $K$-algebra $C$, let us write $\left(C \otimes_{K} K_{n}\right)$ for what we called $\tilde{C}_{n}$ in Definition 1.13. This more closely parallels our notation for schemes. That is, for $Z=\operatorname{Spec} C$, then $\left(Z \times_{K} \operatorname{Spec} K_{n}\right)=\operatorname{Spec}\left(\left(C \otimes_{K} K_{n}\right)^{r}\right)$.

Everything reduces to showing that, for all $Y=\operatorname{Spec} B$, the following are isomorphic.

$$
\left(\left(B \otimes_{K} K_{n}\right) \otimes_{K} K_{m}\right) \cong\left(\left(B \otimes_{K} K_{m}\right) \otimes_{K} K_{n}\right)^{\sim}
$$

Let us write $K_{m}=K[t] /\left(t^{m+1}\right), K_{n}=K[u] /\left(u^{n+1}\right)$, and use $e$ for the twisted map from $K$ to $K_{n}$.

Note that the 'trivial' map

$$
\phi:\left(\left(B \otimes_{K} K_{n}\right) \otimes_{K} K_{m}\right) \longrightarrow\left(\left(B \otimes_{K} K_{m}\right) \otimes_{K} K_{n}\right)
$$

that sends

$$
(1 \otimes f(t) \otimes g(u)) \mapsto(1 \otimes g(u) \otimes f(t))
$$

is not well-defined. For example, for $c \in K$, in $\left(\left(B \otimes_{K} K_{n}\right) \otimes_{K} K_{m}\right)$,

$$
(1 \otimes 1 \otimes c)=(1 \otimes e(c) \otimes 1)
$$

yet, in $\left(\left(B \otimes_{K} K_{m}\right) \otimes_{K} K_{n}\right)$,

$$
\phi(1 \otimes 1 \otimes c) \neq \phi(1 \otimes e(c) \otimes 1) .
$$

But a slight variation of this map does work.

First we claim that any non-zero element of $\left(\left(B \otimes_{K} K_{n}\right) \otimes_{K} K_{m}\right)$ can be written uniquely as a sum, $\sum_{i \leq m, j \leq n}\left(b_{i j} \otimes u^{j} \otimes t^{i}\right)$. Clearly, it suffices to prove this for elements of the form $\left(b \otimes a_{1} u^{j} \otimes a_{2} t^{i}\right)$. And we see that

$$
\begin{aligned}
\left(b \otimes a_{1} u^{j} \otimes a_{2} t^{i}\right) & =\left(b \otimes e\left(a_{2}\right) a_{1} u^{j} \otimes t^{i}\right) \\
=\sum_{k \leq n}\left(b \otimes D_{k}\left(a_{2}\right) a_{1} u^{j+k} \otimes t^{i}\right) & =\sum_{k \leq n}\left(D_{k}\left(a_{2}\right) a_{1} b \otimes u^{j+k} \otimes t^{i}\right),
\end{aligned}
$$


as desired. Uniqueness is obvious. Next we observe that this also holds in the algebra $\left(\left(B \otimes_{K}\right.\right.$ $\left.\left.K_{m}\right) \otimes_{K} K_{n}\right)$. Note that $\left(b \otimes a_{1} t^{i} \otimes a_{2} u^{j}\right) \in\left(\left(B \otimes_{K} K_{m}\right) \otimes_{K} K_{n}\right)$ equals $\left(a_{1} a_{2} b \otimes t^{i} \otimes u^{j}\right)$.

Define

$$
\theta:\left(\left(B \otimes_{K} K_{n}\right) \otimes_{K} K_{m}\right) \longrightarrow\left(\left(B \otimes_{K} K_{m}\right) \otimes_{K} K_{n}\right)
$$

by $\theta\left(b \otimes u^{j} \otimes t^{i}\right)=\left(b \otimes t^{i} \otimes u^{j}\right)$. Clearly, $\theta$ is a ring homomorphism and injective, but we need to show that it is $K$-linear and surjective. (This sounds completely obvious, but the ${ }^{\sim}$ s make this more subtle than it first appears.) Let $c \in K,\left(b \otimes u^{j} \otimes t^{i}\right) \in\left(\left(B \otimes_{K} K_{n}\right) \otimes_{K} K_{m}\right)$. Then

$$
c \cdot\left(b \otimes u^{j} \otimes t^{i}\right)=\left(b \otimes u^{j} \otimes c t^{i}\right)=\sum_{k \leq n}\left(D_{k}(c) b \otimes u^{j+k} \otimes t^{i}\right)
$$

and

$$
\begin{aligned}
& \theta\left(\sum_{k \leq n}\left(D_{k}(c) b \otimes u^{j+k} \otimes t^{i}\right)\right)=\sum_{k \leq n}\left(D_{k}(c) b \otimes t^{i} \otimes u^{j+k}\right) \\
= & \sum_{k \leq n}\left(b \otimes t^{i} \otimes D_{k}(c) u^{j+k}\right)=\left(b \otimes t^{i} \otimes e(c) u^{j}\right) \\
= & c \cdot\left(b \otimes t^{i} \otimes u^{j}\right) .
\end{aligned}
$$

This proves $K$-linearity.

To prove that $\theta$ is surjective, it will suffice to show that for all $c \in K$, that $(1 \otimes 1 \otimes c) \in$ $\left(\left(B \otimes_{K} K_{m}\right) \otimes_{K} K_{n}\right)$ is in the image of $\theta$. The rest then follows easily. By Proposition 1.14, we can rewrite $c$ as $c=\sum_{k \leq n} e\left(c_{k}\right) u^{k}$, so we get that

$$
(1 \otimes 1 \otimes c)=\left(1 \otimes 1 \otimes \sum_{k \leq n} e\left(c_{k}\right) u^{k}\right)=\sum_{k \leq n}\left(e\left(c_{k}\right) \otimes 1 \otimes u^{k}\right) .
$$

Thus $\theta\left(\sum_{k \leq n}\left(e\left(c_{k}\right) \otimes u^{k} \otimes 1\right)\right)=(1 \otimes 1 \otimes c)$.

For completeness, we mention the following, which can be proved in the same way as the previous theorem.

Theorem 2.7. Let $X$ be a $K$-scheme, $m, n \leq \infty$. Then

$$
P_{m}\left(P_{n}(X)\right)=P_{n}\left(P_{m}(X)\right) .
$$

Remark 2.8. Let $X$ be a $K$-scheme. For $0 \leq m \leq n \leq \infty$, the maps $f_{m n}: \operatorname{HS}_{A /(K, \underline{D})}^{m} \rightarrow \operatorname{HS}_{A /(K, \underline{D})}^{n}$ of Remark 1.10, (3) give rise to morphisms

$$
f_{m n}: \operatorname{HS}_{X /(K, \underline{D})}^{m} \longrightarrow \mathrm{HS}_{X /(K, \underline{D})}^{n}
$$

which again form a directed system.

In terms of schemes, the $f_{m n}$ give morphisms

$$
\pi_{n m}: P_{n}(X /(K, \underline{D})) \longrightarrow P_{m}(X /(K, \underline{D}))
$$

which also form a directed system. By Remark 1.10.(3),

$$
\mathrm{HS}_{X /(K, \underline{D})}^{\infty}=\lim _{\overrightarrow{i \in \mathbb{N}}} \mathrm{HS}_{X /(K, \underline{D})}^{i}
$$

and

$$
P_{\infty}(X /(K, \underline{D}))=\lim _{i \in \mathbb{N}} P_{i}(X /(K, \underline{D}))
$$


Functorial properties. There are numerous easy to verify functorial properties of these constructions, exactly as in Voj06. We only mention a few here. Some more general results hold.

For all $m, \mathrm{HS}_{A /(K, \underline{D})}^{m}$ is functorial in pairs $(K, \underline{D}) \rightarrow A$, and $\mathrm{HS}_{X /(K, \underline{D})}^{m}$ and $P_{m}(X /(K, \underline{D}))$ are functorial in pairs $X \rightarrow$ Spec $K$. Given a commutative diagram

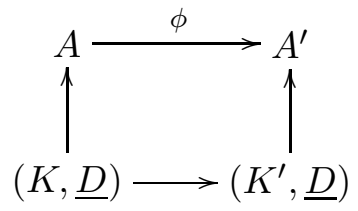

there is an induced commutative diagram

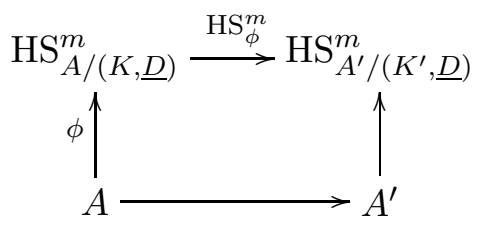

that takes $d_{i} a \in \operatorname{HS}_{A /(K, \underline{D})}^{m}$ to $d_{i} \phi(a) \in \operatorname{HS}_{A^{\prime} /\left(K^{\prime}, \underline{D}\right)}^{m}$ for all $a \in A$ and all $i \leq m$.

Two important cases are base change in $(K, \underline{D})$, and functoriality in $A$, when $K=K^{\prime}$. One also has the following easy lemma.

Lemma 2.9. Let $A$ be a $(K, \underline{D})$-algebra, $\left(K^{\prime}, \underline{D}\right)$ a D-extension field of $K$, and $A^{\prime}=A \otimes_{K} K^{\prime}$. Then $\operatorname{HS}_{A^{\prime} /\left(K^{\prime}, \underline{D}\right)}^{m} \cong \mathrm{HS}_{A /(K, \underline{D})}^{m} \otimes_{K} K^{\prime}$ as $A^{\prime}$-algebras.

Proof. Let $\phi$ be the map from $\operatorname{HS}_{A^{\prime} /\left(K^{\prime}, \underline{D}\right)}^{m}$ to $\operatorname{HS}_{A /(K, \underline{D})}^{m} \otimes_{K} K^{\prime}$ that sends $d_{k}(a \otimes c), k \leq m, a \in$ $A, c \in K$, to $\left.\sum_{i+j=k}\left(d_{i} a \otimes 1\right)\left(1 \otimes D_{j} c\right)\right)$. It is clear that $\phi$ is an isomorphism.

These properties carry over to schemes. The next result is an easy corollary of the above lemma.

Corollary 2.10. Let $(K, \underline{D})$ be a $\mathcal{D}$-field, and let $\left(K^{\prime}, \underline{D}\right)$ be a $\mathcal{D}$-field extension. Then for all $K$-schemes $X$ and all $m$,

$$
P_{m}\left(X \times_{K} \operatorname{Spec} K^{\prime}\right) \cong P_{m}(X) \times_{K} \operatorname{Spec} K^{\prime} .
$$

If $f: X \rightarrow X^{\prime}$ is a morphism of $K$-schemes, one has the following commutative diagram, which lifts $f$ to a map between prolongations.

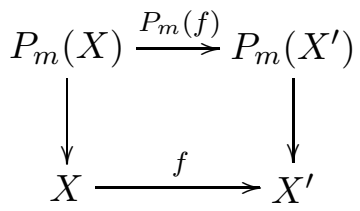

Lemma 2.11. Let $X, X^{\prime}$ be $K$-schemes, and $f: X \rightarrow X^{\prime}$ a closed immersion. Then $P_{m}(f)$ : $P_{m}(X) \rightarrow P_{m}\left(X^{\prime}\right)$ is also a closed immersion.

Proof. It is enough to check locally, on affines, where it follows from Proposition 1.22 .

The following propositions are versions of standard facts about jet spaces, and can be proved in the same way (for example, see [Bli05]).

Proposition 2.12. Let $f: X \rightarrow Y$ be an étale morphism of schemes over a $\mathcal{D}$-field $(K, \underline{D})$. Then for all $m$,

$$
P_{m}(X) \cong X \times_{Y} P_{m}(Y)
$$


Proof. We argue on the corresponding functor of points. For any $K$-scheme $Z$,

$$
\begin{aligned}
\operatorname{Hom}_{K}\left(Z, P_{m}(X)\right) & \simeq \operatorname{Hom}_{K}\left(\left(Z \times_{K} \operatorname{Spec} K_{m}\right), X\right) \\
\operatorname{Hom}_{K}\left(Z, X \times_{Y} P_{m}(Y)\right) & \simeq \operatorname{Hom}_{K}(Z, X) \times_{\operatorname{Hom}_{K}(Z, Y)} \operatorname{Hom}_{K}\left(Z, P_{m}(Y)\right) \\
& \simeq \operatorname{Hom}_{K}(Z, X) \times_{\operatorname{Hom}_{K}(Z, Y)} \operatorname{Hom}_{K}\left(\left(Z \times_{K} \operatorname{Spec} K_{m}\right), Y\right)
\end{aligned}
$$

Consider the following diagram.

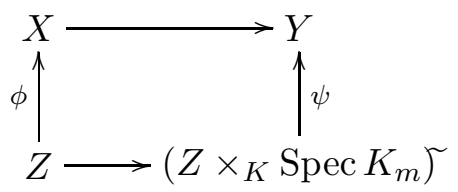

(with a diagonal arrow $\tau$ from $\left(Z \times{ }_{K} \operatorname{Spec} K_{m}\right)$ to $\left.X\right)$. A morphism $\tau$ is a $Z$-valued point of $P_{m}(X)$, and determines a pair of morphisms

$$
(\phi, \psi) \in \operatorname{Hom}_{K}(Z, X) \times_{\operatorname{Hom}_{K}(Z, Y)} \operatorname{Hom}_{K}\left(\left(Z \times_{K} \operatorname{Spec} K_{m}\right), Y\right),
$$

which determines a $Z$-valued point of $X \times_{Y} P_{m}(Y)$. This gives the canonical map from $P_{m}(X)$ to $X \times_{Y} P_{m}(Y)$, which does not depend on any properties of the morphism $f$.

In the other direction, a $Z$-valued point of $X \times_{Y} P_{m}(Y)$ corresponds to a pair of morphisms $(\phi, \psi)$ making the above diagram commute. By formal étaleness, there is a unique $\tau$ completing the diagram. Thus, the map taking $(\phi, \psi)$ to $\tau$ determines the inverse morphism from $X \times_{Y} P_{m}(Y)$ to $P_{m}(X)$, as desired.

Proposition 2.13. Let $X$ be a smooth scheme over the $\mathcal{D}$-field $(K, \underline{D})$ of dimension $n$. Then for all $m \in \mathbb{N}, P_{m}(X)$ is an $\mathbb{A}^{n m}$-bundle over $X$. (That is, $X$ can be covered by open sets $U$ such that $P_{m}(U) \cong U \times_{K} \mathbb{A}^{n m}$.)

Proof. By hypothesis, $X \rightarrow \operatorname{Spec} K$ is a smooth map, so, by [EGA], this implies that there is a covering of $X$ by open sets $U_{i}$, such that for all $i$, the following diagram commutes

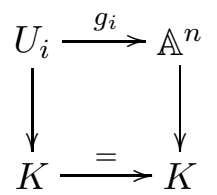

and $g_{i}$ is étale. By the previous proposition, $P_{m}\left(U_{i}\right) \cong U_{i} \times \mathbb{A}^{n m}$, as desired.

By the same argument, one also gets the following.

Corollary 2.14. Let $X$ be an $n$-dimensional smooth scheme over the $\mathcal{D}$-field $(K, \underline{D})$. Then for all $m, P_{m+1}(X)$ is an $\mathbb{A}^{n}$-bundle over $P_{m}(X)$.

\section{D-Schemes}

Many of the definitions and results in this section are from [Bui93].

Definition 3.1. Let $(K, \underline{D})$ be a $\mathcal{D}$-field. A $\mathcal{D}$-scheme over $(K, \underline{D})$ is a $K$-scheme $X$ such that $\mathcal{O}_{X}$ is a structure sheaf of $\mathcal{D}$ - $(K, \underline{D})$-algebras. A morphism of $\mathcal{D}$-schemes is a morphism of $K$-schemes such that the map $\mathcal{O}_{Y} \rightarrow f_{*} \mathcal{O}_{X}$ is a map of sheaves of $\mathcal{D}$ - $(K, \underline{D})$-algebras.

Example 3.2. Let $X$ be a $K$-scheme. Then $P_{\infty}(X)$ is a $\mathcal{D}$-scheme. Given a morphism $f: X \rightarrow Y$ of $K$-schemes, the induced map $f_{\infty}: P_{\infty}(X) \rightarrow P_{\infty}(Y)$ is a morphism of $\mathcal{D}$-schemes. 
Proposition 3.3. Let $(A, \underline{D})$ be an $\mathcal{D}-(K, \underline{D})$-algebra. There exists a $\mathcal{D}$-scheme $X=\mathcal{D}$-Spec $(A, \underline{D})$ such that, forgetting the $\mathcal{D}$-structure on $X, X$ is isomorphic to $\operatorname{Spec} A$.

Proof. To show that one can add a $\mathcal{D}$-structure to $\operatorname{Spec} A$, it suffices to show that the localization of a $\mathcal{D}$-ring is itself a $\mathcal{D}$-ring. This is the content of Lemma 1.7.

One also has the following $\mathcal{D}$-version of a well-known fact from algebraic geometry. (See [Har77], II. Ex. 2.4 and Prop. II.2.3, or [EH00] Thm. I-40.)

Proposition 3.4. Let $(A, \underline{D})$ be a $\mathcal{D}$-ring, and $\left(X, \mathcal{O}_{X}\right)$ a $\mathcal{D}$-scheme. Then there is a bijection:

$$
\chi: \operatorname{Hom}_{\mathcal{D}-\operatorname{Sch}}(X, \operatorname{Spec} A) \longrightarrow \operatorname{Hom}_{\mathcal{D}-\operatorname{Ring}}\left(A, \Gamma\left(X, \mathcal{O}_{X}\right)\right) .
$$

Proof. In the usual case, given a morphism $f: X \rightarrow \operatorname{Spec} A$, and the associated map $f^{\#}: \mathcal{O}_{\operatorname{Spec} A} \rightarrow$ $f_{*} \mathcal{O}_{X}$, one gets a homomorphism $A \rightarrow \Gamma\left(X, \mathcal{O}_{X}\right)$ by taking global sections. This gives a bijection

$$
\chi: \operatorname{Hom}_{\operatorname{Sch}}(X, \operatorname{Spec} A) \longrightarrow \operatorname{Hom}_{R i n g s}\left(A, \Gamma\left(X, \mathcal{O}_{X}\right)\right) .
$$

By definition, if $f: X \rightarrow \mathcal{D}$-Spec $A$ is a $\mathcal{D}$-morphism, then the induced homomorphism $A \longrightarrow$ $\Gamma\left(X, \mathcal{O}_{X}\right)$ is a homomorphism of $\mathcal{D}$-rings, so one has an injection:

$$
\chi_{\mathcal{D}}: \operatorname{Hom}_{\mathcal{D}-\operatorname{Sch}}(X, \mathcal{D}-\operatorname{Spec} A) \longrightarrow \operatorname{Hom}_{\mathcal{D}-\operatorname{Ring}}\left(A, \Gamma\left(X, \mathcal{O}_{X}\right)\right) .
$$

To verify surjectivity, it suffices to look carefully at the construction of $\chi^{-1}$ in [EH].

Remark 3.5. Let $X \subseteq \mathbb{A}^{n}$ be an affine $K$-scheme, $\Gamma\left(X, \mathcal{O}_{X}\right)=K\left[x_{i}\right]_{i=1, \ldots, n} /\left(f_{j}\right)_{j \in J}$. For all $m$, $P_{m}(X)$ is the closed subscheme of $\mathbb{A}^{n m}=\operatorname{Spec}\left(K\left[d_{k} x_{i}\right]_{i=1, \ldots, n, k=0, \ldots, m}\right)$ with

$$
\Gamma\left(P_{m}(X), \mathcal{O}_{P_{m}(X)}\right)=K\left[d_{k} x_{i}\right]_{i=1, \ldots, n, k=0, \ldots, m} /\left(d_{k} f_{j}\right)_{j \in J, k=0, \ldots, m} .
$$

(This follows from Proposition 1.24.) In particular, for every closed point $\left(a_{1}, \ldots, a_{n}\right) \in X$, the point $\left(D_{k} a_{i}\right)_{i=1, \ldots, n, k=0, \ldots, m}$ is in $P_{m}(X)$. The canonical projection from $P_{m}(X)$ to $X$ maps a closed point $\left(a_{i k}\right)_{i=1, \ldots, n, k=0, \ldots, m}$ to its first $n$ coordinates, $\left(a_{i 0}\right)_{i=1, \ldots, n}$.

Next, we define $\mathcal{D}$-polynomial maps between schemes, which we use to define a section of the canonical map $\pi_{m}: P_{m}(X) \rightarrow X$.

Proposition 3.6. Let $(K, \underline{D})$ be a $\mathcal{D}$-field. The prolongation functor, that takes a $K$-scheme $X$ to the $\mathcal{D}$-scheme $P_{\infty}(X)$, is the right adjoint to the forgetful functor $Y \mapsto Y^{!}$from $\mathcal{D}$-schemes to $K$-schemes.

Proof. In [Bui93], p. 1405. This also follows easily from Proposition 1.21.

Recall that given a $K$-scheme $X$, a $K$-rational point of $X$ is a $K$-scheme homomorphism from Spec $K$ to $X$. Likewise, if $X$ is a $\mathcal{D}$-scheme, we will say that a $K$-rational point of $X$ is a $\mathcal{D}$-scheme homomorphism from $\mathcal{D}$-Spec $K$ to $X$. Of course, a $\mathcal{D}$-morphism $f: X \rightarrow Y$ naturally induces a map between their $K$-rational points. The previous proposition immediately implies that there is a natural bijection between $K$-rational points of $X$ and of $P_{\infty}(X)$.

Definition 3.7. Let $X, Y$ be $K$-schemes, and $f: P_{\infty}(X) \rightarrow P_{\infty}(Y)$ be a $\mathcal{D}$-morphism. The natural bijections

and

$$
\chi: \operatorname{Hom}_{K}(\operatorname{Spec} K, X) \longrightarrow \operatorname{Hom}_{(K, \underline{D})}\left(\mathcal{D}-\operatorname{Spec} K, P_{\infty}(X)\right)
$$

$$
\zeta: \operatorname{Hom}_{K}(\operatorname{Spec} K, Y) \longrightarrow \operatorname{Hom}_{(K, \underline{D})}\left(\mathcal{D}-\operatorname{Spec} K, P_{\infty}(Y)\right)
$$

and the induced map

$$
\hat{f}: \operatorname{Hom}_{(K, \underline{D})}\left(\mathcal{D}-\operatorname{Spec} K, P_{\infty}(X)\right) \longrightarrow \operatorname{Hom}_{(K, \underline{D})}\left(\mathcal{D}-\operatorname{Spec} K, P_{\infty}(Y)\right)
$$

determine a (set theoretic) map from $K$-rational points of $X$ to those of $Y$, given by $\zeta^{-1} \circ \hat{f} \circ \chi$. 
A $\mathcal{D}$-polynomial map from $X$ to $Y$ is a map on $K$-rational points of the form $\zeta^{-1} \circ \hat{f} \circ \chi$, for some $\mathcal{D}$-morphism $f: P_{\infty}(X) \rightarrow P_{\infty}(Y)$.

Schemes $X$ and $Y$ are $\mathcal{D}$-polynomially isomorphic if there are $\mathcal{D}$-polynomial maps $f: X \rightarrow Y$ and $g: Y \rightarrow X$ such that $g \circ f=\operatorname{Id}_{X}$ and $f \circ g=\operatorname{Id}_{Y}$.

Remark 3.8. Let $X=\operatorname{Spec}\left(K\left[x_{i}\right]_{i \leq n} /\left(f_{j}\right)_{j \in J}\right)$, so that

$$
P_{\infty}(X)=\operatorname{Spec}\left(K\left[d_{k} x_{i}\right]_{i \leq n, k<\infty} /\left(d_{k} f_{j}\right)_{j \in J, k<\infty}\right) .
$$

The bijection $\chi$ takes $h \in \operatorname{Hom}_{K}(\operatorname{Spec} K, X)$, which is determined by $b_{i}=h\left(x_{i}\right), i \leq n$, to $H \in$ $\operatorname{Hom}_{(K, \underline{D})}\left(\operatorname{Spec} K, P_{\infty}(X)\right)$ determined by $D_{k} b_{i}=H\left(d_{k} x_{i}\right), i \leq n$.

Proposition 3.9. Let $X$ be a $K$-scheme, and $m<\infty$. There exists a D-polynomial map $\nabla_{m}$ : $X \rightarrow P_{m}(X)$ that is a section of the canonical projection $p_{m}: P_{m}(X) \rightarrow X$.

Let $f: X \rightarrow Y$ be a morphism of $K$-schemes. Considering $f$ and $P_{m}(f)$ as maps on $K$-rational points, the following diagram commutes.

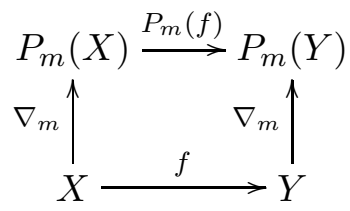

Proof. By the adjointness of $P_{\infty}(-)$ and $(-)^{!}$, there is a natural bijection

$$
\operatorname{Hom}_{K}\left(\left(P_{\infty}(X)\right)^{!}, P_{m}(X)\right) \simeq \operatorname{Hom}_{(K, \underline{D})}\left(P_{\infty}(X), P_{\infty}\left(P_{m}(X)\right)\right) .
$$

Let $f: P_{\infty}(X) \rightarrow P_{\infty}\left(P_{m}(X)\right)$ be the $\mathcal{D}$-morphism corresponding to the canonical projection from $\left(P_{\infty}(X)\right)^{!}$to $P_{m}(X)$, and let $\nabla_{m}$ be the $\mathcal{D}$-polynomial map corresponding to $f$. We show that $\nabla_{m}$ has the desired properties.

It suffices to check locally, so suppose that $X$ is given as $\operatorname{Spec}\left(K\left[x_{i}\right]_{i \leq n} /\left(f_{j}\right)_{j \in J}\right)$. By Remark 3.5.

$$
\begin{aligned}
P_{m}(X) & =\operatorname{Spec}\left(K\left[d_{k} x_{i}\right]_{k \leq m, i \leq n} /\left(d_{k} f_{j}\right)_{k \leq m, j \in J}\right) \\
P_{\infty}(X) & =\operatorname{Spec}\left(K\left[d_{k} x_{i}\right]_{k<\infty, i \leq n} /\left(d_{k} f_{j}\right)_{k<\infty, j \in J}\right) \\
P_{\infty}\left(P_{m}(X)\right) & =\operatorname{Spec}\left(K\left[d_{l} d_{k} x_{i}\right]_{i \leq n, k \leq m, l<\infty} /\left(g_{h}\right)_{h \in H}\right)
\end{aligned}
$$

where $\left(g_{h}\right)_{h \in H}$ is the ideal generated by $\left(d_{l} d_{k} f_{j}\right)_{j \in J, k \leq m, l<\infty}$. The $\mathcal{D}$-morphism from $P_{\infty}(X)$ to $P_{\infty}\left(P_{m}(X)\right)$, corresponding to the projection morphism from $P_{\infty}(X)$ to $P_{m}(X)$ is determined by the $\mathcal{D}$-algebra homomorphism

$$
K\left[d_{l} d_{k} x_{i}\right]_{i \leq n, k \leq m, l<\infty} /\left(g_{h}\right)_{h \in H} \longrightarrow K\left[d_{k} x_{i}\right]_{k<\infty, i \leq n} /\left(d_{k} f_{j}\right)_{k<\infty, j \in J}
$$

that sends $d_{l} d_{k} x_{i}$ to $\left(\begin{array}{c}k+l \\ k\end{array}\right) d_{k+l} x_{i}$. One can then see that this determines the $\mathcal{D}$-polynomial map from $X$ to $P_{m}(X)$ that takes the closed point $\left(a_{i}\right)_{i \leq n}$ to $\left(D_{k} a_{i}\right)_{i \leq n, k \leq m}$. By Remark 3.5, this is a section of $\pi_{m}$.

Next we argue that $P_{m}(f) \circ \nabla_{X}=\nabla_{Y} \circ f$. Again, it suffices to prove this for affine schemes, so assume that $X=\operatorname{Spec} K[\bar{x}] / I$ and $Y=\operatorname{Spec} K[\bar{y}] / J$. Let $S=K[\bar{x}] / I$ and $R=K[\bar{y}] / J$, and let $f$ also denote the homomorphism from $R$ to $S$ corresponding to $f: X \rightarrow Y$. A $K$-rational point of $X$ corresponds to a homomorphism $g$ from $S$ to $K$, which is determined by the image of $\bar{x}$, so we think of a $K$-rational point as a tuple $\bar{a}=g(\bar{x})$ of elements of $K$. Also $P_{m}(X)=\operatorname{Spec~HS}_{S /(K, \underline{D})}^{m}$ is affine, and $\operatorname{HS}_{S /(K, \underline{D})}^{m}$ is generated by $\left(d_{k} x\right)_{x \in \bar{x}, k \leq m}$. We saw above that

$$
\nabla_{X}(\bar{a})=\left(\bar{a}, D_{1}(\bar{a}), \ldots, D_{m}(\bar{a})\right) .
$$


More precisely, $\nabla_{X}(\bar{a})$ is the $K$-rational point of $P_{m}(X)$ that corresponds to the map that sends $d_{k} x \in \operatorname{HS}_{S /(K, \underline{D})}^{m}$ to $D_{k}(g(x)) \in K$, for $x \in \bar{x}$.

Let $f(\bar{a})=\bar{b} \in Y, \bar{b}=(g \circ f(y))_{y \in \bar{y}}$. As above, $\nabla_{Y}(\bar{b})=\left(\bar{b}, D_{1}(\bar{b}), \ldots, D_{m}(\bar{b})\right)$. As a map of $K$-algebras, $P_{m}(f)$ sends $d_{k} y$ to $d_{k} f(y)$, for $y \in \bar{y}, k \leq m$. Thus,

$$
P_{m}(f)\left(\bar{a}, D_{1}(\bar{a}), \ldots, D_{m}(\bar{a})\right)=\left(\bar{b}, D_{1}(\bar{b}), \ldots, D_{m}(\bar{b})\right)
$$

as desired.

The following result is new. It generalizes the well-known fact that the first prolongation of a variety is differentially isomorphic to the tangent space. The standard proof is geometric, using the existence, for any variety $X$, of a differential section $\nabla: X \rightarrow P_{1}(X)$, and fact that $P_{1}(X)$ is a $T X$-torsor. In contrast, our proof below is completely algebraic, though in remarks after the proof we try to provide some geometric intuition. (Recall that, in general for $m>1, J_{m}(X)$ is not a group scheme over $X$, so $P_{m}(X)$ is not a torsor under $J_{m}(X)$. Thus one cannot generalize the standard proof.)

Theorem 3.10. Let $X$ be a $K$-scheme.

(1) $P_{\infty}\left(P_{m}(X)\right)$ and $P_{\infty}\left(J_{m}(X)\right)$ are isomorphic as $\mathcal{D}$-schemes.

(2) $P_{m}(X)$ and $J_{m}(X)$ are $\mathcal{D}$-polynomially isomorphic.

Proof. Part (2) follows immediately from (1), so it suffices to prove (1). We first establish this for affine schemes. The general argument follows by gluing.

Let $X=\operatorname{Spec} A$. Note that

$$
P_{\infty}\left(P_{m}(X)\right)=\operatorname{Spec}\left(\operatorname{HS}_{\mathrm{HS}_{A /(K, \underline{D})}^{\infty}}^{\infty} /(K, \underline{D})\right)
$$

and

$$
P_{\infty}\left(J_{m}(X)\right)=\operatorname{Spec}\left(\operatorname{HS}_{\mathrm{HS}_{A / K}^{m} /(K, \underline{D})}^{\infty}\right) .
$$

Thus we must show that $\mathrm{HS}_{\mathrm{HS}_{A /(K, \underline{D})}^{m} /(K, \underline{D})}^{\infty}$ and $\mathrm{HS}_{\mathrm{HS}_{A / K}^{m} /(K, \underline{D})}^{\infty}$ are isomorphic as $\mathcal{D}$ - $(K, \underline{D})$-algebras. Therefore, the theorem follows from the following proposition.

Proposition 3.11. Let $A$ be a $(K, \underline{D})$-algebra. Then

$$
\left(\mathrm{HS}_{\mathrm{HS}}^{\infty}{ }_{A /(K, \underline{D})}^{m} /(K, \underline{D}), \underline{D}\right) \cong\left(\mathrm{HS}_{\mathrm{HS}_{A / K}^{m} /(K, \underline{D})}^{\infty}, \underline{D}\right) \text {. }
$$

Proof of Proposition. We first treat the case $A$ a polynomial ring, $A=K[\bar{x}]$, where $\bar{x}$ is a (possibly infinite) tuple. Write

$$
\begin{aligned}
R & :=\mathrm{HS}_{\mathrm{HS}_{A /(K, \underline{D})}^{m} /(K, \underline{D})}^{\infty} \cong K\left[d_{i} \delta_{j} x\right]_{0 \leq i<\infty, 0 \leq j \leq m, x \in \bar{x}} \\
S & :=\mathrm{HS}_{\mathrm{HS}_{A / K}^{m} /(K, \underline{D})}^{\infty} \cong K\left[d_{i} \partial_{j} x\right]_{0 \leq i<\infty, 0 \leq j \leq m, x \in \bar{x}} .
\end{aligned}
$$

(Note that $d_{i} \delta_{j} x$ and $d_{i} \partial_{j} x$ are individual symbols. One could just have well written instead $x_{i j}$, but the chosen notation is more suggestive. Below, we often write $\delta_{j} x$, or $\partial_{j} x$, for $d_{0} \delta_{j} x$, or $d_{0} \partial_{j} x$, since we are thinking of $d_{0}$ as the 'identity map.') Observe that $R$ and $S$ are also $\mathcal{D}$-rings, letting $D_{l}\left(d_{i} \delta_{j} x\right)=\left(\begin{array}{c}i+l \\ l\end{array}\right) d_{i+l} \delta_{j} x$ in $R$, likewise for $S$. We often write $D_{i} \partial_{j} x$, or $D_{i} \delta_{j} x$, for $d_{i} \partial_{j} x$, or $d_{i} \delta_{j} x$.

These rings are obviously isomorphic, but we want to construct an isomorphism that we can also use in the general case, $B=K[\bar{x}] / I, I$ any ideal.

Let $\phi: R \rightarrow S$ be the $K$-algebra homomorphism determined by setting

$$
\phi\left(d_{i} \delta_{j} x\right)=D_{i}\left(\sum_{k+l=j} d_{k} \partial_{l} x\right)
$$


for all $i, j$, and $x$. (Of course, $\phi(c)=c$, for $c \in K$.) Moreover, it is clear that $\phi$ is actually a $\mathcal{D}-(K, \underline{D})$-algebra homomorphism.

To prove that $\phi$ is an isomorphism, we define a homomorphism $\psi: S \rightarrow R$ and show that they are inverses of each other. Let $\psi$ be the homomorphism determined by, for all $i, j$, and $x$,

$$
\psi\left(d_{i} \partial_{j} x\right)=D_{i}\left(\sum_{k+l=j}(-1)^{k} D_{k} \delta_{l} x\right)
$$

As above, one sees easily that $\psi$ is also a $\mathcal{D}$ - $(K, \underline{D})$-algebra homomorphism.

First, we show that $\psi \circ \phi=\operatorname{Id}_{R}$. It suffices to calculate this on the generators, $d_{i} \delta_{j} x$.

$$
\begin{aligned}
\psi \circ \phi\left(d_{i} \delta_{j} x\right) & =\psi\left(D_{i} \sum_{k+l=j} d_{k} \partial_{l} x\right) \\
& =D_{i}\left(\sum_{k+l=j} \psi\left(d_{k} \partial_{l} x\right)\right) \\
& =D_{i}\left(\sum_{k+l=j} D_{k}\left(\sum_{a+b=l}(-1)^{a} D_{a} \delta_{b} x\right)\right) \\
& =D_{i}\left(\delta_{j} x\right)+D_{i}\left(\sum_{b=0}^{j-1}\left(\sum_{a+k=j-b}(-1)^{a} D_{a} D_{k} \delta_{b} x\right)\right) \\
& =d_{i} \delta_{j} x+D_{i}\left(\sum_{b=0}^{j-1}\left(\sum_{a+k=j-b}(-1)^{a}\left(\begin{array}{c}
j-b \\
a
\end{array}\right) D_{j-b} \delta_{b} x\right)\right) \\
& =d_{i} \delta_{j} x
\end{aligned}
$$

using the identity $(1-1)^{n}=\sum_{i=0}^{n}(-1)^{i}\left(\begin{array}{c}n \\ i\end{array}\right)=0$, for $n=j-b$. Next, we show that $\phi \circ \psi=\operatorname{Id}_{S}$, arguing again only on generators.

$$
\begin{aligned}
\phi \circ \psi\left(d_{i} \partial_{j} x\right) & =\phi\left(D_{i} \sum_{k+l=j}(-1)^{k} D_{k} \delta_{l} x\right) \\
& =D_{i}\left(\sum_{k+l=j}(-1)^{k} D_{k} \phi\left(\delta_{l} x\right)\right) \\
& =D_{i}\left(\sum_{k+l=j}(-1)^{k} D_{k} \sum_{a+b=l} D_{a} \partial_{b} x\right) \\
& =D_{i}\left(\partial_{j} x\right)+\left(\sum_{b=0}^{j-1} \sum_{k+a=j-b}(-1)^{k} D_{k} D_{a} \partial_{b} x\right) \\
& =d_{i} \partial_{j} x+\left(\sum_{b=0}^{j-1} \sum_{k=0}^{j-b}(-1)^{k}\left(\begin{array}{c}
j-b \\
k
\end{array}\right) \partial_{b} x\right) \\
& =d_{i} \partial_{j} x
\end{aligned}
$$

This completes the proof for $A$ a polynomial ring. We now consider the general case $B$ a $K$ algebra, $B=K[\bar{x}] / I, I$ an ideal. By Corollary 1.24 and the analogous result in Vojta, one gets the following description of $U:=\operatorname{HS}_{\mathrm{HS}_{B /(K, \underline{D})}^{m} /(K, \underline{D})}^{\infty}$. (We change the notation slightly, adding $d_{0}$ and $\partial_{0}$ as 'identity functions.')

$$
U \cong K\left[d_{i} \delta_{j} x\right]_{0 \leq i, 0 \leq j \leq m, x \in \bar{x}} /\left(d_{i} \delta_{j} f\right)_{0 \leq i, 0 \leq j \leq m, f \in I}
$$


For a polynomial $h \in K[\bar{x}]$, the expression $d_{i} \delta_{j} h$ should be considered shorthand for an element of the polynomial ring $K\left[d_{i} \delta_{j} x\right]_{0 \leq i, 0 \leq j \leq m, x \in \bar{x}}$, that can be specified inductively as follows.

$$
\begin{aligned}
& d_{i} \delta_{j} c=\left(\begin{array}{c}
i+j \\
i
\end{array}\right) D_{i+j}(c) \text { for } c \in K \\
& d_{i} \delta_{j}(f+g)=d_{i} \delta_{j} f+d_{i} \delta_{j} g \\
& d_{i} \delta_{j}(f g)=\sum_{s+t=i}\left(\sum_{k+l=j}\left(d_{s} \delta_{k} f\right)\left(d_{t} \delta_{l} g\right)\right) .
\end{aligned}
$$

Likewise, we get the following description of $V:=\operatorname{HS}_{\mathrm{HS}_{B / K}^{m} /(K, \underline{D})}^{\infty}$.

$$
V \cong K\left[d_{i} \partial_{j} x\right]_{0 \leq i, 0 \leq j \leq m, x \in \bar{x}} /\left(d_{i} \partial_{j} f\right)_{0 \leq i, 0 \leq j \leq m, f \in I}
$$

In $V, d_{i} \delta_{j} h$ is defined as in $U$, except that for $c \in K, d_{i} \partial_{j} c=0$ for $j>0$, and $d_{i} \partial_{j} c=D_{i} c$ for $j=0$.

Rings $U$ and $V$ are quotients of $R$ and $S$, defined above, $U=R / J$ and $V=S / L$, where $J$ and $L$ are the ideals from the definitions of $U$ and $V$. We claim that the isomorphism $\phi$ from $R$ to $S$ naturally induces an isomorphism from $U$ to $V$. To prove this, it will suffice to show that $\phi(J) \subseteq L$ and $\psi(L) \subseteq J$. This follows immediately from the next two claims.

Claim 1. For each polynomial $h \in A$ and $d_{i} \delta_{j} h \in R, \phi\left(d_{i} \delta_{j} h\right)=D_{i}\left(\sum_{k+l=j} d_{k} \partial_{l} h\right)$.

Claim 2. For each polynomial $h \in A$ and $d_{i} \partial_{j} h \in S, \psi\left(d_{i} \partial_{j} h\right)=D_{i}\left(\sum_{k+l=j}(-1)^{k} D_{k} \delta_{l} h\right)$.

Proof of Claim 1. Since all maps being considered are additive, it suffices to consider the case $h$ a monomial, $h=a \bar{y}, a \in K$ and $\bar{y}=\left(y_{1}, \ldots, y_{n}\right)$. We introduce the following multi-index notation. A multi-index $\alpha,(\beta, \gamma$, etc. $)$ is a sequence of non-negative integers, $\alpha=\left(\alpha_{1}, \ldots, \alpha_{n}\right)$. We say that the length of $\alpha$ is $n$, and write $\sum \alpha$ for $\sum_{i} \alpha_{i}$.

Using the (generalized) product rule, we can now give an explicit definition, in $R$, of

$$
d_{i} \delta_{j} a \bar{y}:=D_{i}\left(\sum_{k+l=j} \delta_{k} a \delta_{l} \bar{y}\right)=D_{i}\left(\sum_{k+l=j} D_{k} a \cdot\left(\sum_{|\alpha|=n, \sum \alpha=l} \delta_{\alpha_{1}} y_{1} \cdot \ldots \cdot \delta_{\alpha_{n}} y_{n}\right)\right)
$$

Likewise, there is an analogous definition for elements of the ring $S$.

$$
d_{i} \delta_{j} a \bar{y}:=D_{i}\left(a \cdot\left(\sum_{|\alpha|=n, \sum \alpha=j} \delta_{\alpha_{1}} y_{1} \cdot \ldots \cdot \delta_{\alpha_{n}} y_{n}\right)\right)
$$

We now calculate $\phi\left(d_{i} \delta_{j} h\right)$ and $D_{i}\left(\sum_{k+l=j} d_{k} \partial_{l} h\right)$ to show they are equal. Thus

$$
\begin{aligned}
& \phi\left(D_{i} \delta_{j} a \bar{y}\right) \\
= & \phi\left(D_{i}\left(\sum_{k=0}^{j} D_{k} a \cdot\left(\sum_{|\alpha|=n, \sum \alpha=j-k} \delta_{\alpha_{1}} y_{1} \cdot \ldots \cdot \delta_{\alpha_{n}} y_{n}\right)\right)\right) \\
= & D_{i}\left(\sum_{k=0}^{j} D_{k} a \cdot\left(\sum_{|\alpha|=n, \sum \alpha=j-k} \phi\left(\delta_{\alpha_{1}} y_{1} \cdot \ldots \cdot \delta_{\alpha_{n}} y_{n}\right)\right)\right) \\
= & D_{i}\left(\sum_{k=0}^{j} D_{k} a \cdot\left(\sum_{|\beta|=|\gamma|=n, \sum \beta+\sum \gamma=j-k} d_{\beta_{1}} \partial_{\gamma_{1}} y_{1} \cdot \ldots \cdot d_{\beta_{n}} \partial_{\gamma_{n}} y_{n}\right)\right)
\end{aligned}
$$


and

$$
\begin{aligned}
& D_{i}\left(\sum_{l+m=j} d_{l} \partial_{m} a \bar{y}\right) \\
= & D_{i}\left(\sum_{l=0}^{j} D_{l}\left(a \sum_{|\alpha|=n, \sum \alpha=j-l} \partial_{\alpha_{1}} y_{1} \cdot \ldots \cdot \partial_{\alpha_{n}} y_{n}\right)\right) \\
= & D_{i}\left(\sum_{l=0}^{j} \sum_{k+m=l} D_{k} a \cdot\left(D_{m} \sum_{|\alpha|=n, \sum \alpha=j-l} \partial_{\alpha_{1}} y_{1} \cdot \ldots \cdot \partial_{\alpha_{n}} y_{n}\right)\right) \\
= & D_{i}\left(\sum_{k=0}^{j} D_{k} a \cdot\left(\sum_{|\beta|=|\gamma|=n, \sum \beta+\sum \gamma=j-k} d_{\beta_{1}} \partial_{\gamma_{1}} y_{1} \cdot \ldots \cdot d_{\beta_{n}} \partial_{\gamma_{n}} y_{n}\right)\right) .
\end{aligned}
$$

This completes the proof of Claim 1.

Proof of Claim 2. Again we can assume that $h$ is a monomial, $h=a \bar{y}, a \in K$, and $\bar{y}=\left(y_{1}, \ldots, y_{n}\right)$. We want to show that $\psi\left(d_{i} \partial_{j} a \bar{y}\right)$ equals $D_{i} \sum_{k+l=j}(-1)^{k} D_{k} \delta_{l}(a \bar{y})$. We calculate

$$
\begin{aligned}
& \psi\left(d_{i} \partial_{j} a \bar{y}\right) \\
= & a D_{i} \psi\left(\partial_{j} \bar{y}\right)=a D_{i} \psi\left(\sum_{|\alpha|=n, \sum \alpha=j} \delta_{\alpha_{1}} y_{1} \cdot \ldots \cdot \delta_{\alpha_{n}} y_{n}\right) \\
= & a D_{i}\left(\sum_{k+l=j} \sum_{|\beta|=|\gamma|=n, \sum \beta=k \sum \alpha=l}(-1)^{k} d_{\beta_{1}} \delta_{\gamma_{1}} y_{1} \cdot \ldots \cdot d_{\beta_{n}} \delta_{\gamma_{n}} y_{n}\right) \\
= & a D_{i}\left(\sum_{k+l=j}(-1)^{k} D_{k} \delta_{l} \bar{y}\right)
\end{aligned}
$$

and

$$
\begin{aligned}
& D_{i}\left(\sum_{k+l=j}(-1)^{k} D_{k} \delta_{l} a \bar{y}\right) \\
= & D_{i}\left(\sum_{s+t+u+v=j}(-1)^{s+u} D_{s} \delta_{t} a \cdot D_{u} \delta_{v} \bar{y}\right) \\
= & a D_{i}\left(\sum_{u+v=j}(-1)^{u} D_{u} \delta_{v} \bar{y}\right)+D_{i} \sum_{u+v<j}\left(\sum_{s+t=j-(u+v)}(-1)^{s+u} D_{s} \delta_{t} a \cdot D_{u} \delta_{v} \bar{y}\right) \\
= & a D_{i}\left(\sum_{u+v=j}(-1)^{u} D_{u} \delta_{v} \bar{y}\right)+D_{i} \sum_{u+v<j}\left((-1)^{u} \sum_{s+t=j-(u+v)}(-1)^{s}\left(\begin{array}{c}
s+t \\
s
\end{array}\right) D_{s+t} a \cdot D_{u} \delta_{v} \bar{y}\right) \\
= & a D_{i}\left(\sum_{u+v=j}(-1)^{u} D_{u} \delta_{v} \bar{y}\right)+D_{i} \sum_{u+v<j}\left((-1)^{u} \sum_{s=0}^{j-(u+v)}(-1)^{s}\left(\begin{array}{c}
j-(u+v) \\
s
\end{array}\right) D_{j-(u+v)} a \cdot D_{u} \delta_{v} \bar{y}\right) \\
= & a D_{i}\left(\sum_{u+v=j}(-1)^{u} D_{u} \delta_{v} \bar{y}\right) .
\end{aligned}
$$

This completes the proof of Claim 2, and of the Proposition.

This also completes the proof of the Theorem.

Remark 3.12. Our original definition of $\psi$ was

$$
\psi\left(d_{i} \partial_{j} x\right)=D_{i}\left(\sum_{k+l=j} \sum_{\pi \in P[k]}(-i)^{|\pi|} D_{\pi} \delta_{l} x\right)
$$

where $P[k]$ is the set of ordered partitions $\pi$ of $k$, that is, $\pi=\left(a_{1}, \ldots, a_{n}\right) \in\left(\mathbb{N}^{+}\right)^{n}$, with $\sum_{p=1}^{n} a_{p}=$ $k$ and $D_{\pi}=D_{a_{1}} \circ \cdots \circ D_{a_{n}}$. The length of $\pi$ is denoted $|\pi|$. This is the formula one finds if one 
inverts $\phi$ 'by hand' on examples with $i, j$ small. Later, we observed that the following lemma yields the definition that we gave above,

$$
\psi\left(d_{i} \partial_{j} x\right)=D_{i}\left(\sum_{k+l=j}(-1)^{k} D_{k} \delta_{l} x\right) .
$$

Definition 3.13. Given $k \in \mathbb{N}^{+}$, define a function, 'multinomial', $\mu: P[k] \rightarrow \mathbb{N}^{+}$, by

$$
\mu\left(a_{1}, \ldots, a_{n}\right)=\left(\begin{array}{c}
k \\
a_{1}, \ldots, a_{n}
\end{array}\right):=\frac{k !}{a_{1} ! \cdot \ldots \cdot a_{n} !} .
$$

One can easily check that $D_{\pi}=\mu(\pi) D_{k}$.

Lemma 3.14. For all $k \in \mathbb{N}^{+}$,

$$
\sum_{\pi \in P[k]}(-1)^{|\pi|} \mu(\pi)=(-1)^{k}
$$

Proof. By induction on $k$. It will be helpful to stipulate that $P[0]:=\{\emptyset\},|\emptyset|=0$, and $\mu(\emptyset)=1$. The case $k=1$ is obvious, so assume the lemma holds up to $k-1$.

Given a partition $\pi \in P[j], \pi=\left(a_{1}, \ldots, a_{m}\right)$, and $i \in \mathbb{N}^{+}$, let $\pi *(i)$ denote the partition $\left(a_{1}, \ldots, a_{m}, i\right) \in P[j+i]$. Note that $\mu(\pi *(i))=\left(\begin{array}{c}j+i \\ i\end{array}\right) \mu(\pi)$.

$$
\begin{aligned}
& \sum_{\pi \in P[k]}(-1)^{|\pi|}=\sum_{i=1}^{k} \sum_{\pi \in P[k-i]}(-1)^{|\pi|+1} \mu(\pi *(i)) \\
& =-\sum_{i=1}^{k}\left(\begin{array}{c}
k \\
i
\end{array}\right) \sum_{\pi \in P[k-i]}(-1)^{|\pi|} \mu(\pi)=-\sum_{i=1}^{k}\left(\begin{array}{c}
k \\
i
\end{array}\right)(-1)^{k-i} \\
& =\quad(-1)^{k+1} \sum_{i=1}^{k}\left(\begin{array}{c}
k \\
i
\end{array}\right)(-1)^{i}=(-1)^{k}
\end{aligned}
$$

Remark 3.15. We now explain the geometric intuition behind the proof of the preceding proposition. Recall that by the characterizations of jet spaces and prolongations via representable functors, we have the following natural bijections,

$$
\begin{aligned}
& \operatorname{Hom}_{K}\left(\operatorname{Spec} K, J_{m}(X)\right) \simeq \operatorname{Hom}_{K}\left(\operatorname{Spec} K_{m}, X\right) \\
& \operatorname{Hom}_{K}\left(\operatorname{Spec} K, P_{m}(X)\right) \simeq \operatorname{Hom}_{K}\left(\operatorname{Spec} \tilde{K}_{m}, X\right)
\end{aligned}
$$

where, for example, $\operatorname{Hom}_{K}\left(\operatorname{Spec} K, J_{m}(X)\right)$ is the set of $K$-rational points of $J_{m}(X)$. By Proposition 1.14, there is an isomorphism $\Psi$ from $K_{m}$ to $\tilde{K}_{m}$ so there is a corresponding isomorphism $\Psi^{\prime}$ from $\operatorname{Spec} \tilde{K}_{m}$ to Spec $K_{m}$. Thus, $\Psi^{\prime}$ induces a natural bijection from $\operatorname{Hom}_{K}\left(\operatorname{Spec} K_{m}, X\right)$ to $\operatorname{Hom}_{K}\left(\operatorname{Spec} \tilde{K}_{m}, X\right)$, and thus between the $K$-points of $J_{m}(X)$ and $P_{m}(X)$. When one computes this map in local coordinates, one gets the morphisms from the proof of the preceding theorem.

We illustrate this for $X=\mathbb{A}^{1}=\operatorname{Spec} K[x]$. First, we reformulate everything in terms of $K$ algebras. We have $J_{m}(X)=\operatorname{Spec} K\left[\partial_{0} x, \ldots, \partial_{m} x\right]$ and $P_{m}(X)=\operatorname{Spec} K\left[\delta_{0} x, \ldots, \delta_{m} x\right]$ and the following bijections.

$$
\begin{aligned}
\operatorname{Hom}_{K}\left(K\left[\partial_{0} x, \ldots, \partial_{m} x\right], K\right) & \simeq \operatorname{Hom}_{K}\left(K[x], K_{m}\right) \\
\operatorname{Hom}_{K}\left(K\left[\delta_{0} x, \ldots, \delta_{m} x\right], K\right) & \simeq \operatorname{Hom}_{K}\left(K[x], \tilde{K}_{m}\right)
\end{aligned}
$$

An $m$-jet, $\left(a_{0}, \ldots, a_{m}\right) \in J_{m}(X)$, corresponds to the map

$$
f: x \mapsto a_{0}+a_{1} t+\ldots+a_{m} t^{m} \in \operatorname{Hom}_{K}\left(K[x], K_{m}\right),
$$


which corresponds to the map $F \in \operatorname{Hom}_{K}\left(K\left[\partial_{0} x, \ldots, \partial_{m} x\right], K\right)$, where $F\left(\partial_{i} x\right)=a_{i}$. Composing $f$ with the isomorphism $\Psi: K_{m} \rightarrow \tilde{K}_{m}$, one gets the map

$$
g: x \mapsto a_{0}+\left(D_{0} a_{1}+D_{1} a_{0}\right) t+\ldots+\left(\sum_{j+k=m} D_{j} a_{k}\right) t^{m} \in \operatorname{Hom}_{K}\left(K[x], \tilde{K}_{m}\right),
$$

which corresponds to $G \in \operatorname{Hom}_{K}\left(K\left[\delta_{0} x, \ldots, \delta_{m} x\right], K\right.$, where $G\left(\delta_{i} x\right)=\sum_{j+k=i} D_{j} a_{k}$.

Thus, the bijection above between $K$-points of $J_{m}(X)$ and $P_{m}(X)$ sends $\left(a_{0}, \ldots, a_{m}\right) \in J_{m}(X)$ to $\left(a_{0}, D_{0} a_{1}+D_{1} a_{0}, \ldots, \sum_{j+k=m} D_{j} a_{k}\right) \in P_{m}(X)$. This 'differential map' from $J_{m}(X)$ to $P_{m}(X)$ corresponds to the algebraic morphism from $P_{\infty}\left(J_{m}(X)\right)$ to $P_{\infty}\left(P_{m}(X)\right)$ given in the proof of the above theorem by the map $\phi$.

\section{Multiple DeRivations}

We now develop the theory of prolongations over a differential field with finitely many commuting derivations. In characteristic $p>0$, Okugawa Oku87 developed differential algebra over fields with commuting higher derivations. More recently, differential Galois theory for such fields has been investigated by Matzat and van der Put MvdP03]. Ziegler [Zie03] has shown that the model completion of the theory of $n$ commuting Hasse-Schmidt derivations is a definitional expansion of the theory $\mathrm{SCF}_{p, n}$, the theory of separably closed fields of characteristic $p$ and degree of imperfection $n$. Kolchin [Kol73] considers differential fields with commuting derivations, mostly of characteristic 0. Moosa, Pillay, and Scanlon [MPS07] study the model theory of characteristic 0 differential fields with $n$ commuting derivations. Since we will consider rings (fields) of arbitrary characteristic, the results in this section essentially apply to all of the above contexts.

Of course, it would have been possible to consider multiple derivations from the beginning. But it is easier to see the theory developed for one derivation first. The general theory is then quite similar.

Definition 4.1. For $n \in \mathbb{N}^{+}$, a ring with $n$ commuting (higher) derivations is a ring $R$ and a sequence, $\underline{D}_{1}, \ldots, \underline{D}_{n}$, of iterative derivations on $R, \underline{D}_{i}=\left(D_{i, 0}, D_{i, 1}, \ldots\right)$, for $i \leq n$, such that for all $i, j, k, l, D_{i, k} \circ D_{j, l}=D_{j, l} \circ D_{i, k}$.

We also add symbols for 'mixed' derivatives. An $n$-multi-index $\alpha$ is a sequence $\left(\alpha_{1}, \ldots, \alpha_{n}\right)$ of non-negative integers. For each $n$-multi-index $\alpha$, we add an operator $D_{\alpha}$, such that $D_{\alpha}=$ $D_{1, \alpha_{1}} \circ \ldots \circ D_{n, \alpha_{n}}$. So $D_{i, j}=D_{\alpha}$, where $\alpha$ is the multi-index with a $j$ in the $i^{\text {th }}$ place, and 0's everywhere else.

A ring with $n$ commuting derivations will be written $(R, \underline{D})$ when there no chance of confusion. These will also be called $\mathcal{D}$-rings.

Given an $n$-multi-index $\alpha$, the size of $\alpha$, written $|\alpha|$, is the sum $\alpha_{1}+\ldots+\alpha_{n}$. We will sometimes write $\alpha \leq m$ for $|\alpha| \leq m$. There is also a natural partial order on $n$-multi-indices, where $\alpha \leq \beta$ if and only if for all $i \leq n, \alpha_{i} \leq \beta_{i}$. We also writes $\overline{0}$ for the multi-index that is a sequence of 0 's. Note that $D_{\overline{0}}=\operatorname{Id}_{R}$.

Composition of mixed derivatives is completely determined by the iteration rule for each derivation, and the fact that the derivations commute.

Lemma 4.2. Let $(R, \underline{D})$ be a $\mathcal{D}$-ring, and let $D_{\alpha}, D_{\beta}$ mixed derivatives, $\alpha=\left(\alpha_{1}, \ldots, \alpha_{n}\right)$, $\beta=$ $\left(\beta_{1}, \ldots, \beta_{n}\right)$.

$$
D_{\alpha} \circ D_{\beta}=\left(\begin{array}{c}
\alpha_{1}+\beta_{1} \\
\alpha_{1}
\end{array}\right) \cdots\left(\begin{array}{c}
\alpha_{n}+\beta_{n} \\
\alpha_{n}
\end{array}\right) D_{\alpha+\beta}
$$

One also has the following generalization of the Leibniz Rule. 
Proposition 4.3. Let $(R, \underline{D})$ be a $\mathcal{D}$-ring. Then for any multi-index $\alpha$ and any $a, b \in R$,

$$
D_{\alpha}(a b)=\sum_{\beta+\gamma=\alpha} D_{\beta}(a) D_{\gamma}(b)
$$

Proof.

$$
\begin{aligned}
D_{\alpha}(a b) & =D_{1, \alpha_{1}} \circ \ldots \circ D_{n, \alpha_{n}}(a b) \\
& =D_{1, \alpha_{1}} \circ \cdots \circ D_{n-1, \alpha_{n-1}}\left(\sum_{\beta_{n}+\gamma_{n}=\alpha_{n}} D_{n, \beta_{n}}(a) D_{n, \gamma_{n}}(b)\right) \\
& =\sum_{\beta_{n}+\gamma_{n}=\alpha_{n}}\left(D_{1, \alpha_{1}} \circ \cdots \circ D_{n, \alpha_{n}}\left(D_{n, \beta_{n}}(a) D_{n, \gamma_{n}}(b)\right)\right) \\
& \vdots \\
& =\sum_{\beta_{1}+\gamma_{1}=\alpha_{1}}\left(\cdots\left(\sum_{\beta_{n}+\gamma_{n}=\alpha_{n}}\left(D_{1, \beta_{1}} \circ \cdots \circ D_{n, \beta_{n}}(a) \cdot D_{1, \gamma_{1}} \circ \cdots \circ D_{n, \gamma_{n}}(b)\right)\right)\right) \\
& =\sum_{\beta+\gamma=\alpha} D_{\beta}(a) D_{\gamma}(b)
\end{aligned}
$$

Remark 4.4. One sees this rule, for example, when taking Taylor series of holomorphic functions of $n$ variables. Given functions $f$ and $g$, the Leibniz Rule computes the coefficent of $z^{\alpha}$ in the Taylor series of $f g$ from the coefficients of the Taylor series of $f$ and of $g$.

Commuting derivations behave well under localization.

Lemma 4.5. let $(R, \underline{D})$ be a $\mathcal{D}$-ring, with $n$ commuting derivations, and $S \subseteq R$ a multiplicative subset. Then the unique extensions of each of the derivations on $R$ to $S^{-1} R$ also commute.

Proof. See [ku87, Section 1.6, Corollary 1.

Definition 4.6. Let $(R, \underline{D})$ be a D-ring. Given $(R, \underline{D})$-algebras $f: R \rightarrow A$ and $B$, a higher derivation of order $m$ from $A$ to $B$ over $(R, \underline{D})$ is a set of maps $\left\{D_{\alpha}: \alpha \leq m\right\}$ such that $D_{\overline{0}}$ is an $R$-algebra homomorphism, the $D_{\alpha}$ are (additive) abelian group homomorphisms, and

(1) $D_{\alpha}(f(x))=D_{\overline{0}}\left(f\left(D_{\alpha}(x)\right)\right)$;

(2) (Leibniz Rule) $D_{\alpha}(a b)=\sum_{\beta+\gamma=\alpha} D_{\beta}(a) D_{\gamma}(b)$, for all $a, b, \in A, \alpha \leq m$.

Definition 4.7. Let $(R, \underline{D})$ be a $\mathcal{D}$-ring, $f: R \rightarrow A$ an $(R, \underline{D})$-algebra. Define $\mathrm{HS}_{A /(R, \underline{D})}^{m}$ to be the $A$-algebra that is the quotient of the polynomial algebra $A\left[x^{(\alpha)}\right]_{x \in A, 0 \neq \alpha \leq m}$ by the ideal $I$ generated by:

(1) $(x+y)^{(\alpha)}-x^{(\alpha)}-y^{(\alpha)}: x, y \in A, 0 \neq \alpha \leq m$;

(2) $(x y)^{(\alpha)}-\sum_{\beta+\gamma=\alpha} x^{(\beta)} y^{(\gamma)}: x, y \in A, 0 \neq \alpha \leq m$;

(3) $f(r)^{(\alpha)}-f\left(D_{\alpha}(r)\right): r \in R, 0 \neq \alpha \leq m$.

In $A\left[x^{(\alpha)}\right]$, we identify $x \in A$ with $x^{(\overline{0})}$. There is a universal derivation $\underline{d}=\left\{d_{\alpha}: \alpha \leq m\right\}: A \rightarrow$ $\mathrm{HS}_{A /(R, \underline{D})}^{m}$ such that for $\alpha \leq m$ and $x \in A, d_{\alpha}(x)=x^{(\alpha)}$.

Remark 4.8. As before, for $m=\infty$, because $(R, \underline{D})$ is an iterative $\mathcal{D}$-ring, there is a canonical way to make $\mathrm{HS}_{A /(R, \underline{D})}^{\infty}$ into a $\mathcal{D}-(R, \underline{D})$-algebra.

Extend $\underline{d}: A \rightarrow \mathrm{HS}_{A /(R, \underline{D})}^{\infty}$ to an (iterative) higher derivation on $\operatorname{HS}_{A /(R, \underline{D})}^{\infty}$ by letting

$$
d_{\alpha}\left(x^{(\beta)}\right)=\left(\begin{array}{c}
\alpha_{1}+\beta_{1} \\
\alpha_{1}
\end{array}\right) \cdots\left(\begin{array}{c}
\alpha_{n}+\beta_{n} \\
\alpha_{n}
\end{array}\right) x^{(\alpha+\beta)} .
$$


Definition 4.9. Let $(R, \underline{D})$ be a $\mathcal{D}$-ring with $n$ commuting derivations. Let

$$
R_{m}= \begin{cases}R\left[t_{1}, \ldots, t_{n}\right] /\left(t_{1}, \ldots, t_{n}\right)^{m+1} & \text { for } m<\infty \\ R\left[\left[t_{1}, \ldots, t_{n}\right]\right] & \text { for } m=\infty\end{cases}
$$

For a multi-index $\alpha$, write $t^{\alpha}$ as shorthand for $t_{1}^{\alpha_{1}} \cdots t_{n}^{\alpha_{n}}$. For each $m$, we define the twisted homomorphism $e: R \rightarrow R_{m}$ by

$$
e(r)=\sum_{\alpha \leq m} D_{\alpha}(r) t^{\alpha}
$$

Let $\tilde{R}^{m}$ be the $R$-algebra isomorphic to $R_{m}$ as a ring, and made into an $R$-algebra via the map $e: R \rightarrow R_{m}$.

Likewise, given an $(R, \underline{D})$-algebra $f: R \rightarrow B$, let $B_{m}=B\left[t_{1}, \ldots, t_{n}\right] /\left(t_{1}, \ldots, t_{n}\right)^{m+1}$, for $m<\infty$, and $B_{\infty}=B\left[\left[t_{1}, \ldots, t_{n}\right]\right]$. Define $\tilde{f}: R \rightarrow B_{m}$ by

$$
\tilde{f}(r)=\sum_{\alpha \leq m} f\left(D_{\alpha}(r)\right) t^{\alpha}
$$

and let $\tilde{B}_{m}$ be the $(R, \underline{D})$-algebra that is the $\operatorname{ring} B_{m}$ with the map $\tilde{f}: R \rightarrow B_{m}$.

That $e$ and $\tilde{f}$ are actually homomorphisms follows immediately from the Leibniz Rule. One also has the following converse, whose proof is immediate.

Lemma 4.10. Let $R$ be a ring and let $f: R \rightarrow R_{m}$ be a ring homomorphism, which we write

$$
f(b)=\sum_{\alpha \leq m} f_{\alpha}(b) t^{\alpha}
$$

Suppose that $f_{\overline{0}}=\operatorname{Id}_{R}$. Then the maps $\left\{f_{\alpha}: \alpha \leq m\right\}$ are a higher derivation on $R$.

Proposition 4.11. Let $(R, \underline{D})$ be a D-ring with $n$ commuting derivations. For all $m, R_{m}$ and $\tilde{R}_{m}$ are isomorphic as $R$-algebras.

Proof. The idea of the proof is the same as for Lemma 1.14. We first treat the case $m<\infty$. Let $\psi: R_{m} \rightarrow \tilde{R}_{m}$ be the map $\psi(r)=e(r)=\sum_{\alpha \leq m} D_{\alpha}(r) t^{\alpha}$, for $r \in R$, and $\psi\left(t_{i}\right)=t_{i}$, for $i \leq m$. This is clearly a homomorphism, so it remains to check that $\psi$ is injective and surjective.

Linearly order the $n$-multi-indices of size $\leq m, \alpha_{1}, \ldots, \alpha_{k}$ such that, for all $i, j \leq k,\left|\alpha_{i}\right|<\left|\alpha_{j}\right|$ implies $i<j$. Let $b \in R_{m}$ be $b=\sum_{\alpha \leq m} b_{\alpha} t^{\alpha}$, and suppose that $\psi(b)=0$. We will show $b=0$ by showing that each $b_{\alpha_{i}}=0$, by induction on $i$.

$$
\begin{aligned}
\psi(b) & =\psi\left(\sum_{\alpha \leq m} b_{\alpha} t^{\alpha}\right) \\
=\sum_{\alpha \leq m} \psi\left(b_{\alpha}\right) t^{\alpha} & =\sum_{\alpha \leq m}\left(\sum_{\beta+\gamma=\alpha} D_{\beta}\left(b_{\gamma}\right) t^{\alpha}\right)
\end{aligned}
$$

By assumption, each coefficient $\sum_{\beta+\gamma=\alpha} D_{\beta}\left(b_{\beta}\right)$ of $t^{\alpha}$ is 0 . For the base case, $\alpha_{1}=\overline{0}$, the constant term, that is, the coefficient of $t^{\overline{0}}$, is $0=D_{\overline{0}}\left(b_{\overline{0}}\right)=b_{\overline{0}}$.

By induction, suppose that for all $j \leq i, b_{\alpha_{j}}=0$. The $t^{\alpha_{i+1}}$ coefficient of $\psi(b)$ is $0=$ $\sum_{\beta+\gamma=\alpha_{i+1}} D_{\beta}\left(b_{\gamma}\right)=D_{\overline{0}}\left(b_{\alpha_{i+1}}\right)=b_{\alpha_{i+1}}$, because for $\beta+\gamma=\alpha_{i+1}$, if $\beta \neq 0$, then $|\gamma|<\alpha_{i+1}$, so $b_{\gamma}=0$, by the induction hypothesis.

To show that $\psi$ is surjective, it suffices to show that for each $r \in R, r \in \tilde{R}_{m}$ is in $\operatorname{Im}(\psi)$. For fixed $r$, we iteratively define a sequence, $c_{0}, c_{1}, \ldots, c_{k}$, of elements of $R_{m}$ with the following properties. One, for all $i \leq k$, the constant term of $\psi\left(c_{i}\right)$, as a polynomial in the $t_{i}$, is $r$. Two, for $i \geq 1$, and $1 \leq j \leq i$, the coefficient of $t^{\alpha_{j}}$ in $\psi\left(c_{i}\right)$ is 0 . Then $\psi\left(c_{m}\right)=r$, as desired. Set $c_{0}=r$. For the iterative step, suppose that $c_{0}, \ldots, c_{i}$ have been defined, and that $\psi\left(c_{i}\right)=r+\sum_{i+l \leq j \leq k} a_{\alpha_{j}} t^{\alpha_{j}}$. Let $c_{i+1}=c_{i}-a_{\alpha_{i+1}} t^{\alpha_{i+1}}$. Clearly, this procedure yields such a sequence. 
For $m=\infty$, given the isomorphisms $\psi_{i}: R_{i} \rightarrow \tilde{R}_{i}, i<\infty$, it suffices to note again that $R_{\infty}$ and $\tilde{R}_{\infty}$ are the inverse limits of $\left\{R_{i}\right\}_{i<\infty}$ and $\left\{\tilde{R}_{i}\right\}_{i<\infty}$, respectively. The required isomorphism $\psi_{\infty}: R_{\infty} \rightarrow \tilde{R}_{\infty}$ also sends $r \in R$ to $e(r)$, and sends each $t_{i}$ to $t_{i}$.

The next two results are proved in the same way as Proposition 1.18 and Lemma 1.19, respectively.

Proposition 4.12. Let $(R, \underline{D})$ be a $\mathcal{D}$-ring, and $R \rightarrow A$ and $R \rightarrow B$ be $(R, \underline{D})$-algebras. Given a higher derivation $\underline{\delta} \in \operatorname{Der}_{(R, \underline{D})}^{m}(A, B)$, there exists a unique $(R, \underline{D})$-algebra homomorphism, $\phi$ : $\mathrm{HS}_{A /(R, \underline{D})}^{m} \rightarrow B$ such that for all $\alpha \leq m, \delta_{\alpha}=\phi \circ d_{\alpha}$. Thus $\operatorname{HS}_{A /(R, \underline{D})}^{m}$ (together with the universal derivation $\left.\underline{d}: A \rightarrow \operatorname{HS}_{A /(R, \underline{D})}^{m}\right)$ represents the functor $\operatorname{Der}_{(R, \underline{D})}^{m}(A,-)$.

Lemma 4.13. Let $(R, \underline{D}), R \rightarrow A, R \rightarrow B$, and $m$ be as above. Given $\underline{\delta} \in \operatorname{Der}_{(R, \underline{D})}^{m}(A, B)$, define a map $\phi=\phi_{\underline{\underline{\delta}}}: A \rightarrow \tilde{B}_{m}$ by $\phi(a)=\sum_{\alpha \leq m} \delta_{\alpha}(a) t^{\alpha}$. Then $\phi \in \operatorname{Hom}_{R}\left(A, \tilde{B}_{m}\right)$ and the map

$$
\underline{\delta} \mapsto \phi_{\underline{\delta}}: \operatorname{Der}_{(R, \underline{D})}^{m}(A, B) \longrightarrow \operatorname{Hom}_{R}\left(A, \tilde{B}_{m}\right)
$$

is a bijection.

The next corollary is the key result in characterizing prolongations in terms of representable functors, as in Buium.

Corollary 4.14. There is a natural bijection

$$
\operatorname{Hom}_{R}\left(\operatorname{HS}_{A /(R, \underline{D})}^{m}, B\right) \longrightarrow \operatorname{Hom}_{R}\left(A, \tilde{B}_{m}\right) .
$$

Proof. Immediate from Proposition 4.12 and Lemma 4.13.

The following results are proved as in the case of a single derivation.

Proposition 4.15. Let $(R, \underline{D})$ be a $\mathcal{D}$-ring, $\operatorname{Alg}_{R}$ be the category of $(R, \underline{D})$-algebras, and $\mathcal{D}-\operatorname{Alg}_{R}$ be the category of $\mathcal{D}-(R, \underline{D})$-algebras. Let $U$ be the forgetful functor $\mathcal{D}-\operatorname{Alg}_{R} \rightarrow \operatorname{Alg}_{R}$. Then the functor $F: \operatorname{Alg}_{R} \rightarrow \mathcal{D}-\operatorname{Alg}_{R}$, sending $A$ to $\mathrm{HS}_{A /(R, \underline{D})}^{\infty}$, is the left adjoint of $U$.

Proposition 4.16 (Second fundamental exact sequence). Let $(R, \underline{D})$ be $a$ D-ring and $R \rightarrow A \rightarrow B$ a sequence of ring homomorphisms. Assume that $A \rightarrow B$ is surjective, and let I be its kernel. Let $J$ be the ideal in $\mathrm{HS}_{A /(R, \underline{D})}^{m}$ generated by $\left\{d_{\alpha} x: \alpha \leq m, x \in I\right\}$. Then the following sequence is exact.

$$
0 \longrightarrow J \longrightarrow \mathrm{HS}_{A /(R, \underline{D})}^{m} \longrightarrow \mathrm{HS}_{B /(R, \underline{D})}^{m} \longrightarrow 0
$$

In the definition of $J$, it suffices to let $x$ vary over a set of generators of $I$.

Proposition 4.17. Let $(R, \underline{D})$ be a D-ring, and $A=R\left[x_{i}\right]_{i \in I}$. Then $\operatorname{HS}_{A /(R, \underline{D})}^{m}$ is the polynomial algebra $A\left[d_{\alpha} x_{i}\right]_{i \in I, 1 \leq \alpha \leq m}$.

Remark 4.18. For $m, n \geq 1$, define $c_{n, m}$ to be the number of $n$-multi-indices of size $\leq m$. Equivalently, $c_{n, m}$ is the number of monomials in $n$ variables of order $\leq m$ or the number of mixed partial derivatives in $n$ variables of total order $\leq m$.

By the previous proposition, given a polynomial ring $A=R\left[x_{1}, \ldots, x_{q}\right]$ over a ring $R$ with $n$ commuting derivations, then $\mathrm{HS}_{A /(R, \underline{D})}^{m}$ is a polynomial ring in $q \cdot c_{n, m}$ indeterminates.

Corollary 4.19. Let $A$ be an $(R, \underline{D})$-algebra, $A \cong R\left[x_{i}\right]_{i \in I} /\left(f_{j}\right)_{j \in J}$. Then

$$
\mathrm{HS}_{A /(R, \underline{D})}^{m} \cong A\left[d_{\alpha} x_{i}\right]_{i \in I, 1 \leq \alpha \leq m} /\left(d_{\alpha} f_{j}\right)_{j \in J, 1 \leq \alpha \leq m} .
$$


4.1. Prolongations. In this section, we generalize the results of Section 2 to fields with many derivations. Almost everything goes through as before. Assume throughout that $(K, \underline{D})$ is a $\mathcal{D}$ field with $n$ commuting derivations.

Lemma 4.20. Let $A$ be $a(K, \underline{D})$-algebra and $S$ a multiplicative subset of $A$. There is an isomorphism

$$
\mathrm{HS}_{A /(K, \underline{D})}^{m} \otimes_{A} S^{-1} A \longrightarrow \mathrm{HS}_{S^{-1} A /(K, \underline{D})}^{m} \cdot
$$

Theorem 4.21. Let $X$ be a $K$-scheme. For all $m$, there exists a sheaf of $\mathcal{O}_{X}$-algebras $\operatorname{HS}_{X /(K, \underline{D})}^{m}$ such that (i) for each open affine $\operatorname{Spec} A \subseteq X$, there is an isomorphism

$$
\phi_{A}: \Gamma\left(\operatorname{Spec} A, \operatorname{HS}_{X /(K, \underline{D})}^{m}\right) \longrightarrow \operatorname{HS}_{A /(K, \underline{D})}^{m}
$$

of $(K, \underline{D})$-algebras, and (ii) the various $\phi_{A}$ are compatible with the localization isomorphism of Lemma 4.20. Moreover, the collection $\left(\left(\operatorname{HS}_{X /(K, \underline{D})}^{m}\right),\left(\phi_{A}\right)_{A}\right)$ is unique.

Definition 4.22. Let $X$ be a $K$-scheme. For all $m$, the $m^{\text {th }}$-prolongation of $X$ is the scheme

$$
P_{m}(X /(K, \underline{D})):=\operatorname{Spec}_{\mathrm{HS}_{X /(K, \underline{D})}^{m}} .
$$

Suppose that $A$ is a $(K, \underline{D})$-algebra. We write $P_{m}(A /(K, \underline{D}))=P_{m}(\operatorname{Spec} A /(K, \underline{D}))$, which equals Spec $\operatorname{HS}_{A /(K, \underline{D})}^{m}$.

We will also write $X^{m}$ or $P_{m}(X)$ for $P_{m}(X /(K, \underline{D}))$.

Recall that for $m<\infty, K_{m}=K\left[t_{1}, \ldots, t_{n}\right] /\left(t_{1}, \ldots, t^{m+1}\right), K_{\infty}=K\left[\left[t_{1}, \ldots t_{n}\right]\right]$, and that $e$ : $K \rightarrow K_{m}$ denotes the twisted homomorphism. We also let $e: \operatorname{Spec} K_{m} \rightarrow \operatorname{Spec} K$ denote the corresponding twisted morphism of schemes. Given a $K$-scheme $Y$, let $\left(Y \times_{K} \operatorname{Spec} K_{m}\right)$ denote the scheme $\left(Y \times_{K}\right.$ Spec $\left.K_{m}\right)$ made into a $K$-scheme via the map e o $p:\left(Y \times_{K} \operatorname{Spec} K_{m}\right) \rightarrow \operatorname{Spec} K$, where $p:\left(Y \times_{K}\right.$ Spec $\left.K_{m}\right) \rightarrow \operatorname{Spec} K_{m}$ is the canonical projection.

Theorem 4.23. Let $X$ be a $K$-scheme. For all $m$, the scheme $P_{m}(X)$ represents the functor from $K$-schemes to sets given by

$$
Y \mapsto \operatorname{Hom}_{K}\left(\left(Y \times_{K} \operatorname{Spec} K_{m}\right), X\right) .
$$

Theorem 4.24 (Moosa, Pillay, and Scanlon). Let $X$ be a $K$-scheme. For all $m, q \leq \infty$,

$$
J_{m}\left(P_{q}(X)\right) \cong P_{q}\left(J_{m}(X)\right) .
$$

Proof. Both proofs of Theorem 2.6 generalize easily. Here we only show how to adapt the second proof. Exactly as before, it suffices to show that for any $K$-algebra $B$, the following are isomorphic.

$$
\left(\left(B \otimes_{K} K_{q}\right) \otimes_{K} K_{m}\right) \cong\left(\left(B \otimes_{K} K_{m}\right) \otimes_{K} K_{q}\right)^{\sim}
$$

where $K_{m}=K\left[t_{1}, \ldots t_{n}\right] /\left(t_{1}, \ldots, t_{n}\right)^{m+1}, K_{q}=K\left[u_{1}, \ldots, u_{n}\right] /\left(u_{1}, \ldots, u_{n}\right)^{q+1}$, and we use $e$ for the twisted map from $K$ to $K_{q}$.

We claim any non-zero element of $\left(\left(B \otimes_{K} K_{q}\right) \otimes_{K} K_{m}\right)$ can be written uniquely as a sum

$$
\sum_{\alpha \leq m, \beta \leq q}\left(b_{\alpha, \beta} \otimes u^{\beta} \otimes t^{\alpha}\right) .
$$

Again, it suffices to prove this for elements of the form $\left(b \otimes a_{1} u^{\beta} \otimes a_{2} t^{\alpha}\right)$. And

$$
\begin{aligned}
\left(b \otimes a_{1} u^{\beta} \otimes a_{2} t^{\alpha}\right) & =\left(b \otimes e\left(a_{2}\right) a_{1} u^{\beta} \otimes t^{\alpha}\right) \\
=\sum_{\gamma \leq q}\left(b \otimes D_{\gamma}\left(a_{2}\right) a_{1} u^{\beta+\gamma} \otimes t^{\alpha}\right) & =\sum_{\gamma \leq q}\left(D_{\gamma}\left(a_{2}\right) a_{1} b \otimes u^{\beta+\gamma} \otimes t^{\alpha}\right)
\end{aligned}
$$

as desired. Secondly, observe that this also holds in $\left(\left(B \otimes_{K} K_{m}\right) \otimes_{K} K_{q}\right)$, as $\left(b \otimes a_{1} t^{\alpha} \otimes a_{2} u^{\beta}\right) \in$ $\left(\left(B \otimes_{K} K_{m}\right) \otimes_{K} K_{q}\right)$ equals $\left(a_{1} a_{2} b \otimes t^{\alpha} \otimes u^{\beta}\right)$. 
Define

$$
\theta:\left(\left(B \otimes_{K} K_{q}\right) \otimes_{K} K_{m}\right) \longrightarrow\left(\left(B \otimes_{K} K_{m}\right) \otimes_{K} K_{q}\right)
$$

by $\theta\left(b \otimes u^{\beta} \otimes t^{\alpha}\right)=\left(b \otimes t^{\alpha} \otimes u^{\beta}\right)$. It suffices to show that $\theta$ is $K$-linear and surjective. Let $c \in K$, $\left(b \otimes u^{\beta} \otimes t^{\alpha}\right) \in\left(\left(B \otimes_{K} K_{q}\right) \otimes_{K} K_{m}\right)$. Then

$$
c \cdot\left(b \otimes u^{\beta} \otimes t^{\alpha}\right)=\sum_{\gamma \leq q}\left(D_{\gamma}(c) b \otimes u^{\beta+\gamma} \otimes t^{\alpha}\right),
$$

and

$$
\begin{aligned}
& \theta\left(\sum_{\gamma \leq q}\left(D \gamma(c) b \otimes u^{\beta+\gamma} \otimes t^{\alpha}\right)\right)=\sum_{\gamma \leq q}\left(D_{\gamma}(c) b \otimes t^{\alpha} \otimes u^{\beta+\gamma}\right) \\
= & \sum_{\gamma \leq q}\left(b \otimes t^{\alpha} \otimes D_{\gamma}(c) u^{\beta+\gamma}\right)=\left(b \otimes t^{\alpha} \otimes e(c) u^{\beta}\right) \\
= & c \cdot\left(b \otimes t^{\alpha} \otimes u^{\beta}\right) .
\end{aligned}
$$

This proves $K$-linearity.

To prove that $\theta$ is surjective, it will suffice to show that for all $c \in K$, that $(1 \otimes 1 \otimes c) \in$ $\left(\left(B \otimes_{K} K_{m}\right) \otimes_{K} K_{q}\right)$ is in the image of $\theta$. The rest follows easily. By Proposition 4.11, we can write $c$ as $c=\sum_{\gamma \leq q} e\left(c_{\gamma}\right) u^{\gamma}$, so we get that

$$
(1 \otimes 1 \otimes c)=\left(1 \otimes 1 \otimes \sum_{\gamma \leq q} e\left(c_{\gamma}\right) u^{\gamma}\right)=\sum_{\gamma \leq q}\left(e\left(c_{\gamma}\right) \otimes 1 \otimes u^{\gamma}\right) .
$$

Thus

$$
\theta\left(\sum_{\gamma \leq q}\left(e\left(c_{\gamma}\right) \otimes u^{\gamma} \otimes 1\right)\right)=(1 \otimes 1 \otimes c)
$$

Remark 4.25. Let $X$ be a $K$-scheme. As in Remark 2.8, for $0 \leq m \leq n \leq \infty$, the canonical maps $f_{m n}: \operatorname{HS}_{A /(K, \underline{D})}^{m} \rightarrow \operatorname{HS}_{A /(K, \underline{D})}^{n}$ determine a directed system of morphisms

$$
f_{m n}: \mathrm{HS}_{X /(K, \underline{D})}^{m} \longrightarrow \mathrm{HS}_{X /(K, \underline{D})}^{n} .
$$

In terms of schemes, the $f_{m n}$ give morphisms

$$
\pi_{n m}: P_{n}(X /(K, \underline{D})) \longrightarrow P_{m}(X /(K, \underline{D}))
$$

which also form a directed system. Exactly as above, we also have

$$
\mathrm{HS}_{X /(K, \underline{D})}^{\infty}=\lim _{\overrightarrow{i \in \mathbb{N}}} \mathrm{HS}_{X /(K, \underline{D})}^{i}
$$

and

$$
P_{\infty}(X /(K, \underline{D}))=\lim _{i \in \mathbb{N}} P_{i}(X /(K, \underline{D}))
$$

Functorial properties. There are many functorial properties of these constructions, precisely as discussed on page 13 .

Lemma 4.26. Let $A$ be a $(K, \underline{D})$-algebra, $\left(K^{\prime}, \underline{D}\right)$ a D-extension field of $K$, and $A^{\prime}=A \otimes_{K} K^{\prime}$. Then $\mathrm{HS}_{A^{\prime} /\left(K^{\prime}, \underline{D}\right)}^{m} \cong \mathrm{HS}_{A /(K, \underline{D})}^{m} \otimes_{K} K^{\prime}$ as $A^{\prime}$-algebras.

Proof. Let $\phi$ be the map from $\mathrm{HS}_{A^{\prime} /\left(K^{\prime}, \underline{D}\right)}^{m}$ to $\operatorname{HS}_{A /(K, \underline{D})}^{m} \otimes_{K} K^{\prime}$ that sends $d_{\alpha}(a \otimes c), \alpha \leq m, a \in$ $A, c \in K$, to $\left.\sum_{\beta+\gamma=k}\left(d_{\beta} a \otimes 1\right)\left(1 \otimes D_{\gamma} c\right)\right)$. It is clear that $\phi$ is an isomorphism. 
Corollary 4.27. Let $(K, \underline{D})$ be a $\mathcal{D}$-field, and let $\left(K^{\prime}, \underline{D}\right)$ be a $\mathcal{D}$-field extension. Then for all $K$-schemes $X$ and all $m$,

$$
P_{m}\left(X \times{ }_{K} \operatorname{Spec} K^{\prime}\right) \cong P_{m}(X) \times_{K} \operatorname{Spec} K^{\prime} .
$$

As above, if $f: X \rightarrow X^{\prime}$ is a morphism of $K$-schemes, then there is an induced map $P_{m}(f)$ : $P_{m}(X) \rightarrow P_{m}\left(X^{\prime}\right)$ between their prolongations.

Lemma 4.28. Let $X, X^{\prime}$ be $K$-schemes, and $f: X \rightarrow X^{\prime}$ a closed immersion. Then $P_{m}(f)$ : $P_{m}(X) \rightarrow P_{m}\left(X^{\prime}\right)$ is also a closed immersion.

Proposition 4.29. Let $f: X \rightarrow Y$ be an étale morphism of schemes over a $\mathcal{D}$-field $(K, \underline{D})$. Then for all $m$,

$$
P_{m}(X) \cong X \times_{Y} P_{m}(Y) .
$$

Remark 4.30. Notice by Remark 4.18 that for any $q$ and any $m<\infty, \operatorname{dim}\left(P_{m}\left(\mathbb{A}^{q}\right)\right)=q \cdot c_{n, m}$.

Proposition 4.31. Let $X$ be a smooth scheme over the $\mathcal{D}$-field $(K, \underline{D})$ of dimension $q$. Then for all $m, P_{m}(X)$ is an $\mathbb{A}^{q \cdot c_{n, m}}$-bundle over $X$. (That is, $X$ can be covered by open sets $U$ such that $P_{m}(U) \cong U \times_{K} \mathbb{A}^{q \cdot c_{n, m}}$.

Proof. By hypothesis, $X \rightarrow \operatorname{Spec} K$ is a smooth map, so, by [EGA], this implies that there is a covering of $X$ by open sets $U_{i}$, such that for all $i$, the following diagram commutes

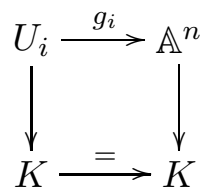

and $g_{i}$ is étale. By the previous proposition, $P_{m}\left(U_{i}\right) \cong U_{i} \times \mathbb{A}^{q \cdot c_{n, m}}$, as desired.

Corollary 4.32. Let $X$ be a smooth scheme over the $\mathcal{D}$-field $(K, \underline{D})$ of dimension $q$. Then for all $m, P_{m+1}(X)$ is an $\mathbb{A}^{q\left(c_{n, m}-c_{n, m-1}\right)}$-bundle over $P_{m}(X)$.

4.2. D-Schemes. We generalize material from Section 3, which is straightforward.

Definition 4.33. Let $(K, \underline{D})$ be a $\mathcal{D}$-field. A $\mathcal{D}$-scheme over $(K, \underline{D})$ is a $K$-scheme $X$ such that $\mathcal{O}_{X}$ is a structure sheaf of $\mathcal{D}$ - $(K, \underline{D})$-algebras. A morphism of $\mathcal{D}$-schemes is a morphism of $R$-schemes such that the map $\mathcal{O}_{Y} \rightarrow f_{*} \mathcal{O}_{X}$ is a map of sheaves of $(R, \underline{D})$-algebras.

Proposition 4.34. Let $(A, \underline{D})$ be a $\mathcal{D}-(K, \underline{D})$-algebra. There exists a $\mathcal{D}$-scheme $X=\mathcal{D}$-Spec $(A, \underline{D})$ such that, forgetting the $\mathcal{D}$-structure on $X, X$ is isomorphic to $\operatorname{Spec} A$.

Proposition 4.35. Let $(A, \underline{D})$ be a $\mathcal{D}$-ring, and $\left(X, \mathcal{O}_{X}\right)$ a $\mathcal{D}$-scheme. Then there is a bijection:

$$
\chi: \operatorname{Hom}_{\mathcal{D}-\operatorname{Sch}}(X, \operatorname{Spec} A) \longrightarrow \operatorname{Hom}_{\mathcal{D}-\operatorname{Ring}}\left(A, \Gamma\left(X, \mathcal{O}_{X}\right)\right) .
$$

Remark 4.36. Let $X \subseteq \mathbb{A}^{q}$ be an affine $K$-scheme, $\Gamma\left(X, \mathcal{O}_{X}\right)=K\left[x_{i}\right]_{i=1, \ldots, q} /\left(f_{j}\right)_{j \in J}$. For all $m \leq \infty, P_{m}(X)$ is the closed subscheme of $\mathbb{A}^{q \cdot c_{n, m}}=\operatorname{Spec}\left(K\left[D_{\alpha} x_{i}\right]_{i=1, \ldots, q, \alpha \leq m}\right)$ with

$$
\Gamma\left(P_{m}(X), \mathcal{O}_{P_{m}(X)}\right)=K\left[D_{\alpha} x_{i}\right]_{i=1, \ldots, q, \alpha \leq m} /\left(D_{\alpha} f_{j}\right)_{j \in J, \alpha \leq m} .
$$

(This follows from Proposition 4.19, In particular, for every closed point $\left(a_{1}, \ldots, a_{q}\right) \in X$, the point $\left(D_{\alpha} a_{i}\right)_{i=1, \ldots, q, \alpha \leq m}$ is in $P_{m}(X)$. The canonical projection from $P_{m}(X)$ to $X$ maps a closed point $\left(a_{i, \alpha}\right)_{i=1, \ldots, q, \alpha \leq m}$ to its first $q$ coordinates, $\left(a_{i, 0}\right)_{i=1, \ldots, q}$.

Proposition 4.37. Let $(K, \underline{D})$ be a $\mathcal{D}$-field. The prolongation functor, that takes a $K$-scheme $X$ to the $\mathcal{D}$-scheme $P_{\infty}(X)$, is the right adjoint to the forgetful functor $Y \mapsto Y^{!}$from $\mathcal{D}$-schemes to K-schemes. 
As before, if $X$ is a $\mathcal{D}$-scheme, we define a $K$-rational point of $X$ to be a $\mathcal{D}$-scheme homomorphism from $\mathcal{D}$-Spec $K$ to $X$. Of course, a D-morphism $f: X \rightarrow Y$ naturally induces a map between their $K$-rational points. The previous proposition immediately implies that there is a natural bijection between $K$-rational points of $X$ and of $P_{\infty}(X)$.

Definition 4.38. Let $X, Y$ be $K$-schemes, and $f: P_{\infty}(X) \rightarrow P_{\infty}(Y)$ be a $\mathcal{D}$-morphism. The natural bijections

$$
\chi: \operatorname{Hom}_{K}(\operatorname{Spec} K, X) \longrightarrow \operatorname{Hom}_{(K, \underline{D})}\left(\mathcal{D}-\operatorname{Spec} K, P_{\infty}(X)\right)
$$

and

$$
\zeta: \operatorname{Hom}_{K}(\operatorname{Spec} K, Y) \longrightarrow \operatorname{Hom}_{(K, \underline{D})}\left(\mathcal{D}-\operatorname{Spec} K, P_{\infty}(Y)\right)
$$

and the induced map

$$
\hat{f}: \operatorname{Hom}_{(K, \underline{D})}\left(\mathcal{D}-\operatorname{Spec} K, P_{\infty}(X)\right) \longrightarrow \operatorname{Hom}_{(K, \underline{D})}\left(\mathcal{D}-\operatorname{Spec} K, P_{\infty}(Y)\right)
$$

determine a (set theoretic) map from $K$-rational points of $X$ to those of $Y$, given by $\zeta^{-1} \circ \hat{f} \circ \chi$.

A D-polynomial map from $X$ to $Y$ is a map on $K$-rational points of the form $\zeta^{-1} \circ \hat{f} \circ \chi$, for some $\mathcal{D}$-morphism $f: P_{\infty}(X) \rightarrow P_{\infty}(Y)$.

Schemes $X$ and $Y$ are $\mathcal{D}$-polynomially isomorphic if there are $\mathcal{D}$-polynomial maps $f: X \rightarrow Y$ and $g: Y \rightarrow X$ such that $g \circ f=\operatorname{Id}_{X}$ and $f \circ g=\operatorname{Id}_{Y}$.

Remark 4.39. Let $X=\operatorname{Spec}\left(K\left[x_{i}\right]_{i \leq q} /\left(f_{j}\right)_{j \in J}\right)$, so that

$$
P_{\infty}(X)=\operatorname{Spec}\left(K\left[d_{\alpha} x_{i}\right]_{i \leq q, \alpha<\infty} /\left(f_{j}\right)_{j \in J}\right) .
$$

The bijection $\chi$ takes $h \in \operatorname{Hom}_{K}(\operatorname{Spec} K, X)$, which is determined by $\left(b_{i}\right)_{i \leq q}=h\left(x_{i}\right)_{i \leq q}$ to $H \in$ $\operatorname{Hom}_{(K, \underline{D})}\left(\operatorname{Spec} K, P_{\infty}(X)\right)$ determined by $\left(D_{\alpha} b_{i}\right)_{i \leq q, \alpha<\infty}=H\left(d_{\alpha} x_{i}\right)_{i \leq q, \alpha<\infty}$.

Proposition 4.40. Let $X$ be a $K$-scheme, and $m<\infty$. There exists a $\mathcal{D}$-polynomial map $\nabla_{m}$ : $X \rightarrow P_{m}(X)$ that is a section of the canonical projection $p_{m}: P_{m}(X) \rightarrow X$.

Let $f: X \rightarrow Y$ be a morphism of $K$-schemes. Considering $f$ and $P_{m}(f)$ as maps on $K$-rational points, the following diagram commutes.

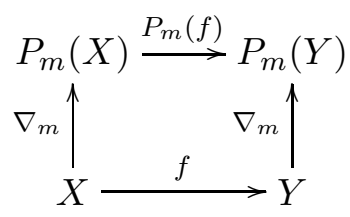

Proof. (Again, the proof is similar to the corresponding result for fields with a single derivation.) By the adjointness of $P_{\infty}(-)$ and $(-)^{!}$, there is a natural bijection

$$
\operatorname{Hom}_{K}\left(\left(P_{\infty}(X)\right)^{!}, P_{m}(X)\right) \simeq \operatorname{Hom}_{(K, \underline{D})}\left(P_{\infty}(X), P_{\infty}\left(P_{m}(X)\right)\right) .
$$

Let $f: P_{\infty}(X) \rightarrow P_{\infty}\left(P_{m}(X)\right)$ be the $\mathcal{D}$-morphism corresponding to the canonical projection from $\left(P_{\infty}(X)\right)^{!}$to $P_{m}(X)$, and let $\nabla_{m}$ be the $\mathcal{D}$-polynomial map corresponding to $f$. We show that $\nabla_{m}$ has the desired properties.

It suffices to check locally, so suppose that $X$ is given as $\operatorname{Spec}\left(K\left[x_{i}\right]_{i \leq q} /\left(f_{j}\right)_{j \in J}\right)$. By Remark 4.36 ,

$$
\begin{aligned}
P_{m}(X) & =\operatorname{Spec}\left(K\left[d_{\alpha} x_{i}\right]_{\alpha \leq m, i \leq q} /\left(d_{\alpha} f_{j}\right)_{\alpha \leq m, j \in J}\right) \\
P_{\infty}(X) & =\operatorname{Spec}\left(K\left[d_{\alpha} x_{i}\right]_{\alpha<\infty, i \leq q} /\left(d_{\alpha} f_{j}\right)_{\alpha<\infty, j \in J}\right) \\
P_{\infty}\left(P_{m}(X)\right) & =\operatorname{Spec}\left(K\left[d_{\beta} d_{\alpha} x_{i}\right]_{\alpha \leq m, \beta<\infty, i \leq q} /\left(g_{h}\right)_{h \in H}\right)
\end{aligned}
$$


where $\left(g_{h}\right)_{h \in H}$ is the ideal generated by $\left(d_{\beta} d_{\alpha} f_{j}\right)_{j \in J, \alpha \leq m, l<\infty}$. The $\mathcal{D}$-morphism from $P_{\infty}(X)$ to $P_{\infty}\left(P_{m}(X)\right)$, corresponding to the projection morphism from $P_{\infty}(X)$ to $P_{m}(X)$ is determined by the $\mathcal{D}$-algebra homomorphism

$$
K\left[d_{\beta} d_{\alpha} x_{i}\right]_{\alpha \leq m, \beta<\infty, i \leq q} /\left(g_{h}\right)_{h \in H} \longrightarrow K\left[d_{\alpha} x_{i}\right]_{\alpha<\infty, i \leq q} /\left(d_{\alpha} f_{j}\right)_{\alpha<\infty, j \in J}
$$

such that

$$
d_{\beta} d_{\alpha} x_{i} \mapsto\left(\begin{array}{c}
\alpha_{1}+\beta_{1} \\
\alpha_{1}
\end{array}\right) \cdots\left(\begin{array}{c}
\alpha_{n}+\beta_{n} \\
\alpha_{n}
\end{array}\right) d_{\alpha+\beta} x_{i} .
$$

One can then see that this determines the $\mathcal{D}$-polynomial map from $X$ to $P_{m}(X)$ that takes the closed point $\left(a_{i}\right)_{i \leq n}$ to $\left(D_{\alpha} a_{i}\right)_{\alpha \leq m, i \leq n}$. By Remark 4.36, this is a section of $\pi_{m}$.

Next we argue that $P_{m}(f) \circ \nabla_{X}=\nabla_{Y} \circ f$. It suffices to prove this for affine schemes, so assume that $X=\operatorname{Spec} K[\bar{x}] / I$ and $Y=\operatorname{Spec} K[\bar{y}] / J$. Let $S=K[\bar{x}] / I$ and $R=K[\bar{y}] / J$, and let $f$ also denote the homomorphism from $R$ to $S$ corresponding to $f: X \rightarrow Y$. A $K$-rational point of $X$ corresponds to a homomorphism $g$ from $S$ to $K$, which is determined by the image of $\bar{x}$, so we think of a $K$-rational point as a tuple $\bar{a}=g(\bar{x})$ of elements of $K$. Also, $P_{m}(X)=\operatorname{Spec~HS}_{S /(K, \underline{D})}^{m}$ is affine, and $\operatorname{HS}_{S /(K, \underline{D})}^{m}$ is generated by $\left(d_{\alpha} x\right)_{x \in \bar{x}, \alpha \leq m}$. We have seen that $\nabla_{X}(\bar{a})=\left(\bar{a}, D_{1}(\bar{a}), \ldots, D_{m}(\bar{a})\right)$. To be more precise, $\nabla_{X}(\bar{a})$ is the $K$-rational point of $P_{m}(X)$ that corresponds to the map that sends $d_{\alpha} x \in \operatorname{HS}_{S /(K, \underline{D})}^{m}$ to $D_{\alpha}(g(x)) \in K$, for each $x \in \bar{x}, \alpha \leq m$.

Let $f(\bar{a})=\bar{b} \in Y, \bar{b}=(g \circ f(y))_{y \in \bar{y}}$. Again, $\nabla_{Y}(\bar{b})=\left(\bar{b}, D_{1}(\bar{b}), \ldots, D_{m}(\bar{b})\right)$. As a map of $K$-algebras, $P_{m}(f)$ is the map that sends $d_{\alpha} y$ to $d_{\alpha} f(y)$, for $y \in \bar{y}, \alpha \leq m$. Thus,

$$
P_{m}(f)\left(\bar{a}, D_{1}(\bar{a}), \ldots, D_{m}(\bar{a})\right)=\left(\bar{b}, D_{1}(\bar{b}), \ldots, D_{m}(\bar{b})\right)
$$

as desired.

\section{REFERENCES}

[Bli05] Manuel Blickle. A short course in geometric motivic integration. Preprint, 2005. arXiv:math. AG/0507404.

[Bui92] Alexandru Buium. Differential algebraic groups of finite dimension, volume 1506 of Lecture Notes in Mathematics. Springer-Verlag, Berlin, 1992.

[Bui93] Alexandru Buium. Geometry of differential polynomial functions. I. Algebraic groups. Amer. J. Math., 115(6):1385-1444, 1993.

[BV95] Alexandru Buium and José Felipe Voloch. Reduction of the Manin map modulo p. J. Reine Angew. Math., 460:117-126, 1995.

[BV96] Alexandru Buium and José Felipe Voloch. Lang's conjecture in characteristic $p$ : an explicit bound. Compositio Math., 103(1):1-6, 1996.

[Cra04] Alastair Craw. An introduction to motivic integration. In Strings and geometry, volume 3 of Clay Math. Proc., pages 203-225. Amer. Math. Soc., Providence, RI, 2004.

[DL99] Jan Denef and François Loeser. Germs of arcs on singular algebraic varieties and motivic integration. Invent. Math., 135(1):201-232, 1999.

[EH00] David Eisenbud and Joe Harris. The geometry of schemes, volume 197 of Graduate Texts in Mathematics. Springer-Verlag, New York, 2000.

[Gil02] Henri Gillet. Differential algebra - a scheme theory approach. In Differential algebra and related topics (Newark, NJ, 2000), pages 95-123. World Sci. Publishing, River Edge, NJ, 2002.

[Har77] Robin Hartshorne. Algebraic geometry. Springer-Verlag, New York, 1977. Graduate Texts in Mathematics, No. 52.

[HP00] Ehud Hrushovski and Anand Pillay. Effective bounds for the number of transcendental points on subvarieties of semi-abelian varieties. Amer. J. Math., 122(3):439-450, 2000.

[Joh85] Joseph Johnson. Prolongations of integral domains. J. Algebra, 94(1):173-210, 1985.

[Kol73] E. R. Kolchin. Differential algebra and algebraic groups. Academic Press, New York, 1973. Pure and Applied Mathematics, Vol. 54.

[Mar06] David Marker. Model theory of differential fields. In Model theory of fields, volume 5 of Lecture Notes in Logic, pages 38-113. Association for Symbolic Logic, La Jolla, CA, second edition, 2006.

[Mat89] Hideyuki Matsumura. Commutative ring theory, volume 8 of Cambridge Studies in Advanced Mathematics. Cambridge University Press, Cambridge, second edition, 1989. Translated from the Japanese by M. Reid. 
[MPS07] Rahim Moosa, Anand Pillay, and Thomas Scanlon. Differential arcs and regular types in differential fields. To appear in J. Reine Angew. Math., 2007.

[MvdP03] B. Heinrich Matzat and Marius van der Put. Iterative differential equations and the Abhyankar conjecture. J. Reine Angew. Math., 557:1-52, 2003.

[Oku87] Kôtaro Okugawa. Differential algebra of nonzero characteristic, volume 16 of Lectures in Mathematics. Kinokuniya Company Ltd., Tokyo, 1987.

[Pil02] Anand Pillay. Differential fields. In Lectures on algebraic model theory, volume 15 of Fields Inst. Monogr., pages 1-45. Amer. Math. Soc., Providence, RI, 2002.

[Pil04] Anand Pillay. Mordell-Lang conjecture for function fields in characteristic zero, revisited. Compos. Math., 140(1):64-68, 2004.

[PZ03] Anand Pillay and Martin Ziegler. Jet spaces of varieties over differential and difference fields. Selecta Math. (N.S.), 9(4):579-599, 2003.

[Sca97] Thomas Scanlon. The abc theorem for commutative algebraic groups in characteristic p. Internat. Math. Res. Notices, (18):881-898, 1997.

[Sca02] Thomas Scanlon. Model theory and differential algebra. In Differential algebra and related topics (Newark, NJ, 2000), pages 125-150. World Sci. Publishing, River Edge, NJ, 2002.

[Voj06] Paul Vojta. Jets via Hasse-Schmidt derivations. In Diophantine Geometry, Proceedings, pages 335-361. Edizioni della Normale, Pisa, 2006.

[Zie03] Martin Ziegler. Separably closed fields with Hasse derivations. J. Symbolic Logic, 68(1):311-318, 2003.

Department of Mathematics, Massachusetts Institute of Technology, 77 Massachusetts Ave., CamBRIDGE, MA 02139

E-mail address: rosen@math.mit.edu

$U R L:$ http://math.mit.edu/ rosen 